\title{
Following the SPARC: tracking Secreted Protein Acidic and Rich in Cysteine associated with LX-2's extracellular vesicles as a non-destructive modality to evaluate lipid-based antifibrotic treatments
}

\section{Cristina Zivko}

University of Bern

Kathrin Fuhrmann

Saarland University

Gregor Fuhrmann

Saarland University

Paola Luciani ( $\sim$ paola.luciani@unibe.ch )

University of Bern https://orcid.org/0000-0001-9664-0149

Article

Keywords: extracellular vesicles, liver fibrosis, proteomics, SPARC, HSC, essential phospholipids, NTA, EAF4, polyenylphosphatidylcholines

Posted Date: November 30th, 2021

DOI: https://doi.org/10.21203/rs.3.rs-1124359/v1

License: (c) (i) This work is licensed under a Creative Commons Attribution 4.0 International License.

Read Full License

Version of Record: A version of this preprint was published at Communications Biology on October 30th, 2022. See the published version at https://doi.org/10.1038/s42003-022-04123-z. 
Following the SPARC: tracking Secreted Protein Acidic and Rich in Cysteine associated with LX-2's extracellular vesicles as a non-destructive modality to evaluate lipid-based antifibrotic treatments

Cristina Zivko ${ }^{1,2,3}$, Kathrin Fuhrmann ${ }^{3}$, Gregor Fuhrmann ${ }^{3,4^{*}}$, Paola Luciani ${ }^{1,2^{*}}$

${ }^{1}$ Institute of Pharmacy, Friedrich Schiller University of Jena, Jena, Germany

2 Department of Chemistry, Biochemistry and Pharmaceutical Sciences, University of Bern, Bern, Switzerland

${ }^{3}$ Helmholtz Institute for Pharmaceutical Research Saarland, Department of Pharmacy, Saarland University, Saarbrücken, Germany

${ }^{4}$ Current address: Department of Biology, Friedrich-Alexander-University Erlangen, Erlangen, Germany

*Email to: gregor.fuhrmann@fau.de; paola.luciani@unibe.ch

Uncovering the complex cellular mechanisms underlying hepatic fibrogenesis, a highly dynamic and active process ultimately responsible for liver failure if left untreated, could expedite the development of effective treatments and noninvasive diagnostic modalities for this often silent pathology. The biochemical complexity of extracellular vesicles (EVs) and their role in intercellular communication make them an attractive tool to look for biomarkers that might become a viable alternative to invasive liver biopsies. We developed a solid set of methods to isolate and characterize EVs from differently treated human hepatic stellate cell (HSC) line LX-2 in vitro, and we investigated the biological effect they exert onto naïve LX-2, proving that EVs do play an active role in fibrogenesis. Electrical/asymmetric flow field-flow fractionation (EAF4) revealed EV subpopulations with different physicochemical behaviors. Proteomic data from our samples was mined for EV-associated proteins whose expression correlated with HSC treatment. Consequently, we chose the secreted protein acidic and cysteine rich (SPARC), a matricellular protein previously reported to be upregulated in activated HSCs, as a proof-of-concept protein to explore the feasibility of using fluorescence nanoparticle tracking analysis as a non-destructive tool for the determination of HSCs' fibrogenic phenotype based on EVs. We could thus use EVs to directly evaluate the efficacy of treatment with S80, a lipid rich (>75 \%) in polyenylphosphatidylcholines (PPC). We found that PPC-rich $\mathbf{S 8 0}$ reduces the relative presence of SPARC-positive EVs. For the first time, we could correlate the cellular response to lipid-based antifibrotic treatment to the relative presence of a candidate protein marker associated with the released EVs. In addition to providing novel insights into PPC treatments, our findings pave the way for more precise and less invasive diagnostic analyses of hepatic fibrogenesis.

Keywords: extracellular vesicles; liver fibrosis; proteomics; SPARC; HSC; essential phospholipids; NTA; EAF4; polyenylphosphatidylcholines 
The liver plays multifaceted roles in many physiologically critical functions: from protein syntheses, to xenobiotic biotransformation, to immunological support ${ }^{1,2}$. Diseases compromising this essential organ directly lead to the death of 2 million people worldwide every year, i.e., $3.5 \%$ of all annual deaths ${ }^{3-5}$. Adequate treatments for liver diseases have yet to be developed to relieve the global health burden arising from hepatic conditions $\mathbf{s}^{6,7}$.

Under physiologically healthy conditions, hepatic stellate cells (HSCs) store vitamin A in cytoplasmic lipid droplets. Upon liver insults, however, HSCs undergo transdifferentiation and become activated, losing their lipid droplets and promoting fibrogenesis ${ }^{8-10}$. In chronic conditions, the escalating deposition of excess, collagen-rich extracellular matrix leads to cirrhosis, and eventually to organ failure ${ }^{11,12}$. The current gold standard for the diagnosis of liver fibrosis is a tissue biopsy, although alternative (albeit imprecise) methods are being explored, such as those based on ultrasonography-methods, as well as those relying on clinical parameters ${ }^{13-17}$. The search for sensitive, precise and non-invasive tools for the evaluation of liver fibrosis and its progression is an open field of investigation. This is particularly important because of liver fibrosis's mostly asymptomatic progression in its early and crucial stages ${ }^{18,19}$.

Extracellular vesicles (EVs) are membranous nanosized vesicles mediating inter-cellular communication ${ }^{20-}$ ${ }^{23}$, and they are increasingly being investigated for their potential as diagnostic tools ${ }^{24-26}$. Methods to work with EVs can be as varied as the research groups devoted to developing them ${ }^{2}$, but the international community has been trying to push for a more rigorous standardization of protocols ${ }^{27}$.

Here we established a robust series of methods for the isolation and thorough characterization of EVs originating from differently treated LX-2 cells, an immortalized human HSC cell line retaining key features of transdifferentiated human $\mathrm{HSCs}^{28}$, building upon an in vitro model of liver fibrosis previously developed in our group ${ }^{29}$. We evaluated the effect of EVs isolated from previously treated HSCs on naïve HSCs. Finally, we mined our EV-associated proteomic data for the development of treatment discriminating tools. We established convenient methods to reliably detect exosomal markers CD81 and CD927and to select cell status-discriminating proteins using fluorescence nanoparticle tracking analysis (f-NTA). We chose the Secreted Protein Acidic and Rich in Cysteine (SPARC), an extracellular matrix (ECM) protein, supported by previous reports on its role in fibrogenic processes ${ }^{30-32}$. SPARC's association with EVs, as shown by our f-NTA method, was then used to evaluate the response of naïve HSC cells to treatment with lipids, most notably soy phosphatidylcholine (SPC), the beneficial effects of which we have reported before. ${ }^{29}$ Essential phospholipids (polyunsaturated phosphatidylcholines (PPC)-enriched soybean extracts) have long been indicated as a supportive therapy for liver diseases even though their mechanism of action is not well understood $^{33-35}$. For the first time, we can not only use biochemical information from EVs to assess the performance of anti-fibrotic PPCs on HSCs, but also to provide novel insights into the role of action of essential phospholipids on HSCs. 


\section{Results}

\section{EV isolation, purification and characterisation.}

To establish the optimal experimental setup (see Scheme 1 in the Methods), EVs from untreated (in Dulbecco's Modified Eagle Medium (DMEM), activated), quiescent-like (treated with retinol, ROL, and palmitic acid, PA) and perpetuated LX-2 cells (treated with transcriptional growth factor $\beta 1$, TGF) were analyzed in terms of yield, size, zeta potential, morphology and protein content. EVs from differently treated LX-2 cells were isolated from serum free conditioned cell culture medium (CCM) after $24 \mathrm{~h}$ of treatment (CCMa, which includes treatment solutions) by differential centrifugation and ultracentrifugation (UC). Subsequently, they were purified by size exclusion chromatography (SEC) (see Methods). Cells were then washed with PBS and given fresh serum-free DMEM; EVs were isolated again after $24 \mathrm{~h}$ (CCMb). Unless otherwise stated, results herein stem from EVs isolated from CCMb on the day of CCM harvest, even though the short-term stability of EVs was tested under different conditions for up to 21-28 d (Fig. S1). The full size distribution profiles of the isolated EVs consistently showed polydisperse populations (Fig. 1a). Quantile subtraction of the distribution curve obtained from untreated cells showed that quiescent LX-2 produce larger EVs (> $100 \mathrm{~nm}$ ) more prominently than TGF-treated cells (Fig. 1b). Scanning electron microscopy (SEM) and transmission electron cryomicroscopy (cryo-TEM) imaging confirmed the polydispersity in the samples (Figs. 1c,d), as well as the expected morphology of the spherical, membrane bound vesicles. After UC, up to $80 \%$ of total particles could be pelleted (Fig. S2). After SEC, EVs were successfully separated from protein aggregates co-purified during UC as determined by bicinchoninic acid (BCA) assay (Fig. 1f-h). Protein content associated with EVs was only detectable after 8 and $9 \mathrm{~mL}$ and was comparable in all groups. TLX-2 treated differently yielded EVs in similar amounts and of similar sizes and zeta potential values (Table S1). Importantly, the documented cell viability at the time of CCMb collection was always above $95 \%$ (Table S1).

EV-containing pellets collected from CCMb from differently treated cells were analyzed by electrical/ asymmetric flow field flow fractionation (EAF4). AF4 technology allows for the unique separation of nanoparticles by two perpendicular flows ${ }^{36,37}$. It has been used with the aim of separating distinctive EVsubpopulations using in vitro and ex vivo samples ${ }^{38,39}$, though not using HSC-EVs. The application of an electrical field in EAF4 approaches opens up the possibility of an in-depth investigation of EV subpopulations based on electrophoretic mobility ${ }^{40-42}$.

Fractionation of our EV pellets after ultracentrifugation of CCMs reveals a broad size distribution of EVs around $300 \mathrm{~nm}$ with rather low electrophoretic mobility of $0.01 \times 10^{-8} \mathrm{~m}^{2} /(\mathrm{V} \cdot \mathrm{s})$ and $1 \times 10^{-8} \mathrm{~m}^{2} /(\mathrm{V} \cdot \mathrm{s})$ for DMEM, TGF and ROL/PA treated cells, respectively. In addition, only the CCMs of untreated and perpetuated cells exhibit another mixed population of larger EVs sterically eluting with smaller EVs (around $1 \mu \mathrm{m}$ and $50 \mathrm{~nm}$, respectively, electrophoretic mobility around $-7 \times 10^{-8} \mathrm{~m}^{2} /(\mathrm{V} \cdot \mathrm{s})$ ). While EVs had similar elution times, there were differences between EV subsets; a later eluting peak was present in all samples (Figs. $1 \mathrm{~h}-\mathrm{j}$ ). For ROL/PA, EVs in this second peak were generally larger compared to their DMEM and TGF counterparts, confirming the subtle size shifts observed when comparing EVs purified by SEC (Figs. 1a,b). Moreover, 
upon applying different electrical fields, a shift for the later eluting population of ROL/PA EVs was noticed, whereas there was no shift noticeable in the fractograms of EVs from DMEM and TGF treated cells. All fractograms of the EVs from the three treatment conditions of the producing cells exhibit a distinct fingerprint. Combined, this information points to the presence of unique EV-subsets, possibly relating to the phenotypical state of the cells from which they were isolated.

a

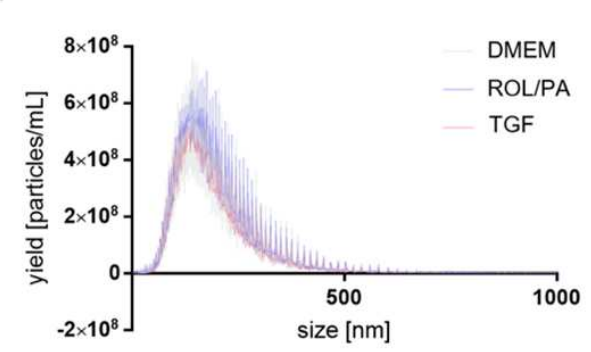

C

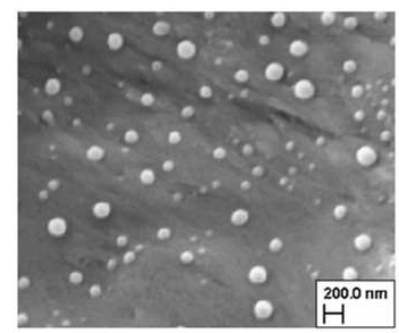

$\mathbf{f}$

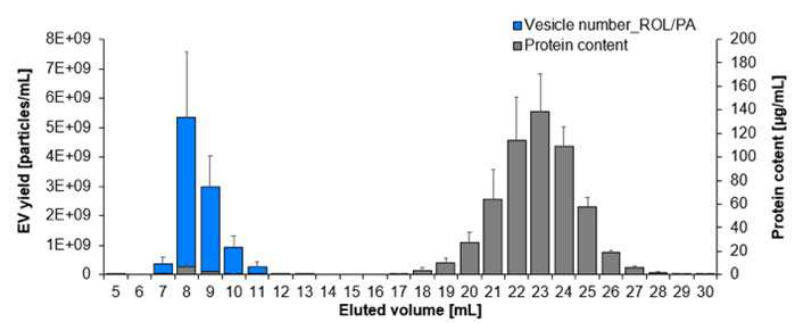

$\mathbf{h}$
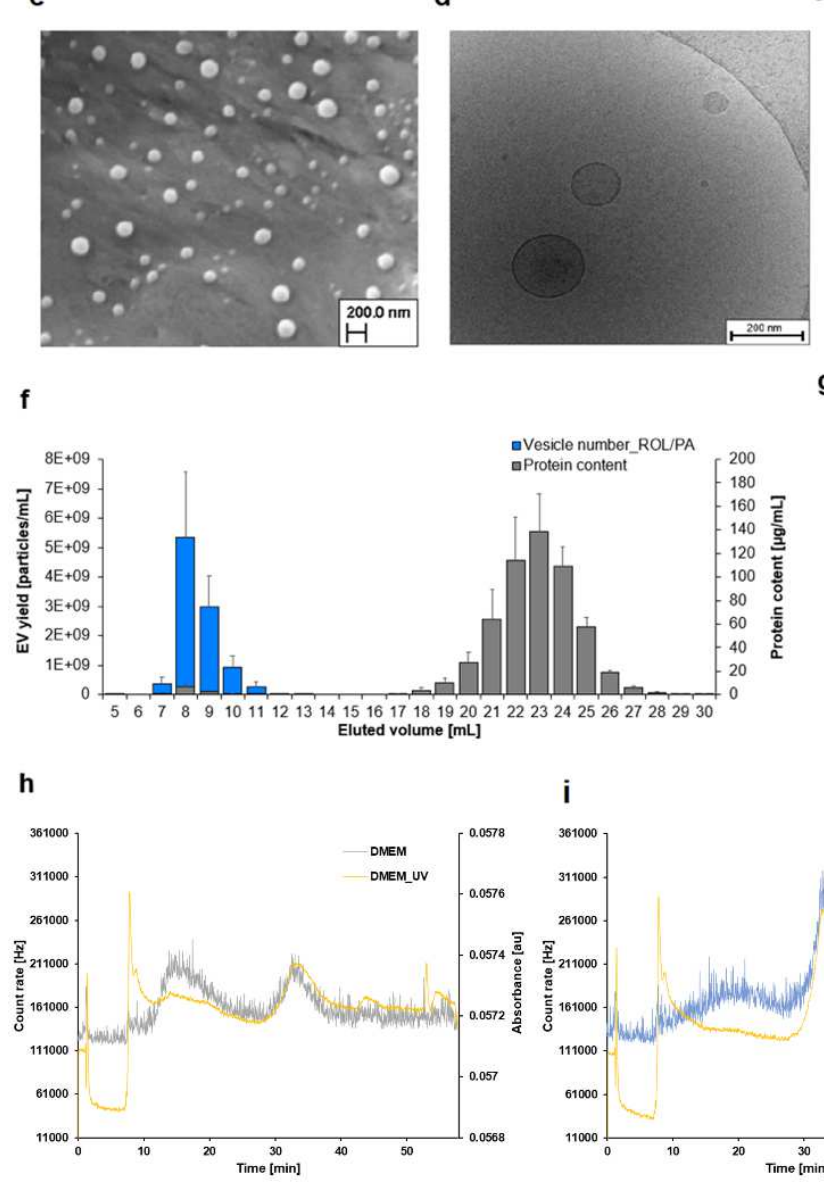

b

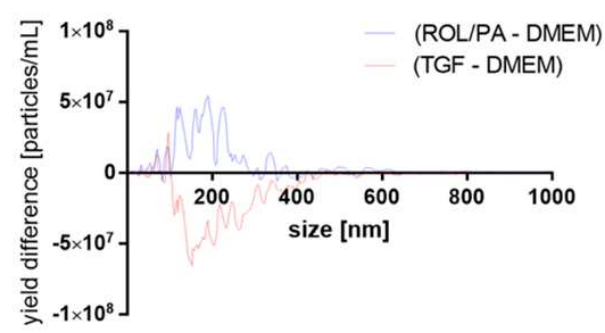

e

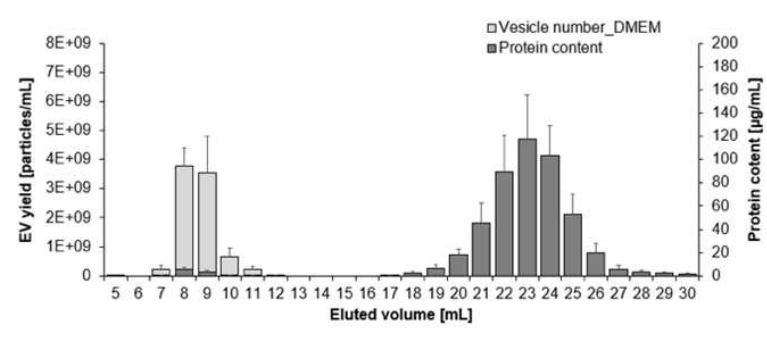

g

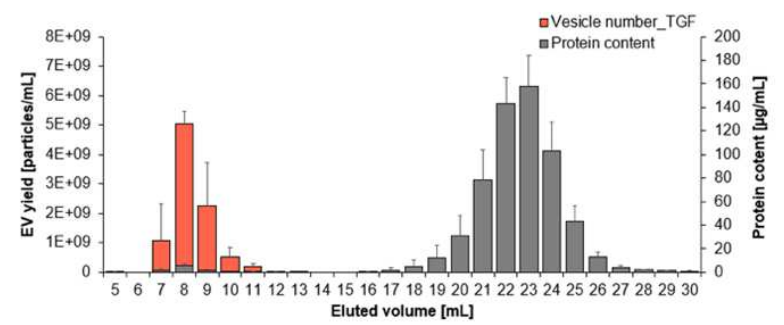
Eluted volume [mL]

j

Fig. 1 | Isolation and characterization of EVs from LX-2 cells. a, Size distribution profiles of EVs isolated from differently treated cells (mean $\pm S D, n=3$ ). $\mathbf{b}$, Quantile subtraction of the yields. c,d, SEM (c) and cryo-TEM (d) images of EVs isolated from untreated cells. e,f,g, Protein content and vesicle number in the collected SEC fractions obtained from untreated $(\mathbf{e})$, quiescent $(\mathbf{f})$ and perpetuated $(\mathbf{g})$ LX-2 (mean \pm SD, $n$ $=3)$. $\mathbf{h}, \mathbf{i}, \mathbf{j}$, Representative EAF4 fractograms of EV collected from untreated (h), quiescent (i) and perpetuated (j). 


\section{Treatment of fresh LX-2 with EV pellets isolated from differently treated LX-2.}

We previously confirmed ${ }^{29}$ that the combination of ROL/PA can deactivate LX-2 cells, and we reported how liposomes containing PPCs showed an even stronger deactivation effect, as seen by the formation of cytoplasmic lipid droplets, which are indicative of the cells being in a quiescent-like status. Oil Red O (ORO) staining was thus performed to reveal the presence of lipid droplets upon different treatments. Confirming our previous results, considerably more lipid droplets were identified in cells treated with $\mathbf{S} 80$ compared to any of the other treatments (Figs. 2a,b). Using EV-pellets from CCMa (i.e., CCM that still includes treatment solution) resulted in trends mirroring those observed by direct treatment (Figs. S4,7). This was true also when treatment using CCMa-EV was followed with treatment with CCMb-EVs (Figs. S5,7). While not as pronounced as the response to direct treatment, residual PPCs from CCMa cannot be excluded as a contributing factor. It is for this reason that the more striking results come from CCMb-EVs (Figs. 2a,b and Figs. S6,7). Fluorescence microscopy images were quantitatively analyzed and used to evaluate cellular response (Fig. 2b). Cells that were treated with EV-containing pellets originating from the CCMb of TGF or S80-treated cells had a response that was strikingly similar to cells that were treated with TGF or S80 directly, albeit to a lesser extent. When looking at the effects of CCMb-TGF on naive cells, the microscopy images show that, remarkably, naïve cells self-assemble along a structured network, much like TGF-treated cells. Functional spatial rearrangement of HSCs has indeed been previously reported ${ }^{43}$. Since the EVs were not purified by SEC in any of these cases, contamination with cellular factors co-purified during UC cannot be excluded. On the other hand, lipid droplets could still be found in cells treated using CCMb-S80 only, even though these cells were never in direct contact with the liposomal formulation, and even though any possible residual S80 from the supernatant of the treated LX-2 was washed away after the collection of CCMa. The newly stored lipid material must have been recycled through EVs released by the originally treated LX-2.

Taken together, these results strongly suggest that EVs from either quiescent-like or perpetuated LX-2 cells might be sufficient to induce a correlated phenotypical status change in otherwise untreated cells. 
a

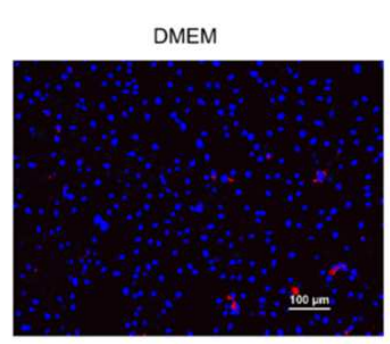

HEPES $10 \%$

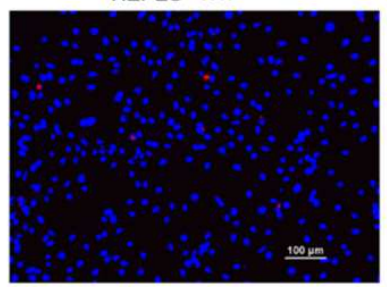

$\mathrm{CCM}(\mathrm{b}) \mathrm{DMEM}$

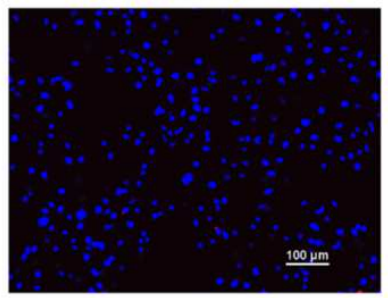

$\mathrm{CCM}(\mathrm{b})$ HEPES

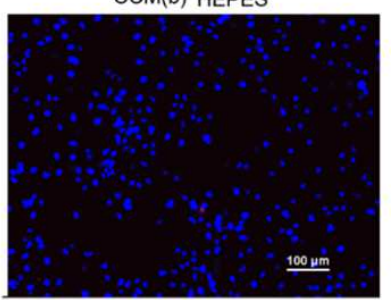

b

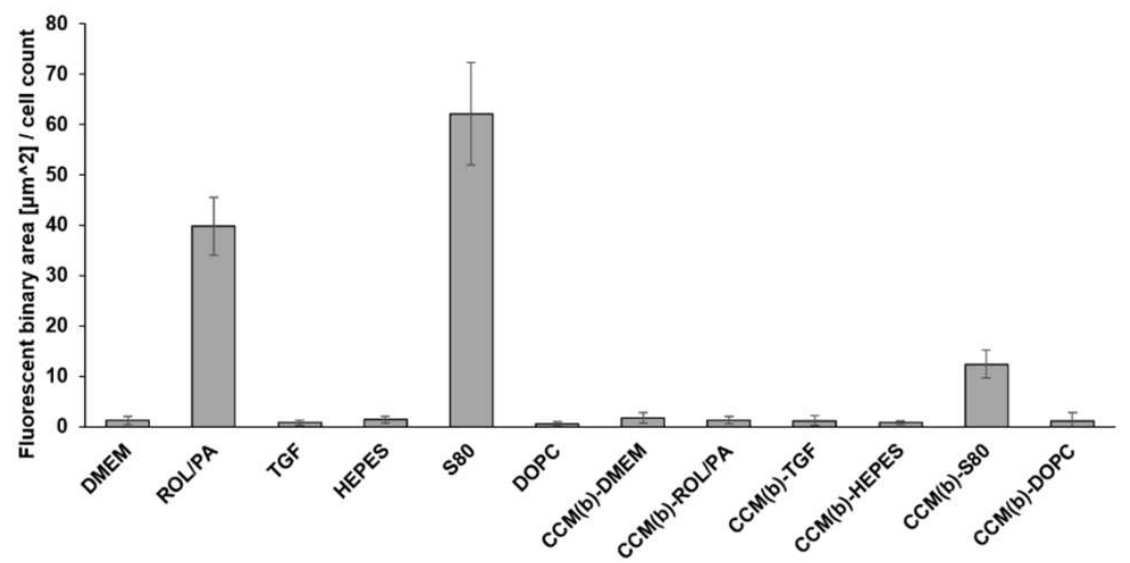

Fig. 2 | Treatment of fresh LX-2 with EV-pellets from previously treated cells. a, Representative images of ORO staining in fluorescence (seen as red spots; nuclei stained with blue DAPI) of differently treated cells after thresholding. b, Quantitative analysis of stained lipid droplets, whereby the fluorescent area (correlating to a quiescent-like status) was normalized to cell count (mean $\pm S D, n=3$ ). 


\section{Proteomic analysis of SEC-purified EVs.}

To further investigate the analytical differences from NTA and EAF4 analyses (Fig. 1), as well as the biological effects exerted by EV-pellets (Fig. 2), we developed methods for the isolation and analysis of EVassociated proteins (see Fig. S8). We hypothesized that purified EVs allow correlation to the physiological state of their cell of origin, as growing evidence from the EV community tends to corroborate ${ }^{44,45}$. Mass spectrometry analysis was carried out on EVs purified by SEC using the peak fraction eluting from 7 to 8 $\mathrm{mL}$ (Fig. 1), leading to the identification of 3,881 unique proteins.

A first examination of the SEC-purified EVs by principal component analysis (PCA) shows the degree of systematic variation in the proteomic profiles among biologically independent samples: EVs originating from similarly treated cells are more similar to each other than to the EVs from any of the other treatment setups (Fig. 3a). Hierarchical clustering of the same samples further demonstrated the similarities within treatment groups (Fig. 3b), as the unsupervised script correctly grouped protein profiles accordingly. Clustering of the proteins on the other axis of the heat map shows two more things. First, there are fundamental similarities across all HSC-EVs, with many protein families shared across samples, especially in the upper third of the heat map. Second, even with all these similarities, the rest of the heat map is characterized by differences in the intensity levels, and by patterns of missing protein hits indicated by white areas.

In a second exploratory step, we generated lists of proteins from all the single hits that would allow more immediate comparisons in a restrictive manner. We decided to consider only proteins which were identified in all single replicates and which could be reliably quantified both by label free quantification (LFQ) and by the sum of the three most intense peptide intensities (Top3) by MaxQuant, referring to them as persistent proteins from here on in. There were 3,388 in total; out of those, 1,931 proteins could be found and quantified in all replicates from all six different treatments (Fig. 3c). This data was mined to confirm the presence of established exosomal markers such as CD81 and CD9 tetraspanins in all samples, as well as the absence of known contaminants such as calnexin ${ }^{27}$.

For every treatment group, there were proteins which were consistently found in addition to the 1,931 proteins that were shared among all. A few of them were also exclusive, i.e., not strictly detected in any of the other groups (Fig. 3c). The thus generated lists of treatment group-specific persistent proteins were all cross-referenced against each other. A summary of the number of proteins found upon every direct comparison is found in Fig. 3d. Volcano plots for every comparison are found in Fig. S8.

Next, results from Welch's t-test were used to look for significant differences of protein recovery levels by group wise comparison of profiles from every condition. This created a new list of 1,146 proteins that were either over or under expressed in at least one of the single comparisons. Cross-referencing this list with the 1,931 persistent proteins that were shared among all treatment groups yielded a panel of 44 proteins (Fig. $3 e$ ). For ease of comparison, a simple, normalized recovery score was developed by adding the LFQ values of every protein for each treatment condition and normalizing them to the sum of LFQ values from each protein, so that the panel could be visually inspected as a heat map (Fig. 3e). It can now be readily seen that TGF-EVs (negative control, indicative of perpetuated LX-2 cells) are more akin to DMEM-EVs than 
they are to ROL/PA-EVs (positive control, indicative of a quiescent-like status). What is even more remarkable is that profibrotic EVs from TGF-treated cells are starkly different to S80-EVs in this rationally designed panel (Fig. 3e). This is not the case for EVs from DOPC-treated cells (negative PPC control to S80), nor for the EVs from the HEPES buffer control. This means that the observed effect is not a result of mere phospholipid treatment, rather, it stems from the combined benefit of specific bioactive, antifibrotic lipids present in S80. We have thus created a screening tool powerful enough to not only distinguish between our three basic controls (DMEM, ROL/PA and TGF), but which also has the potential to semiquantitatively evaluate the performance of additional treatments if further developed. An exploratory Gene Ontology (GO) analysis on the 44-proteins panel from Fig. 3e was performed using the Protein ANalysis THrough Evolutionary Relationships (PANTHER) platform ${ }^{46,47}$. There were no protein groups clearly associated to any specific pathway when taken together, only sparse hits (Fig. 4).

While such a panel of 44 proteins is considerably smaller than a full proteomic dataset, we postulated that there might be a selection of proteins to more simply tell apart quiescent-like LX-2 cells and their perpetuated (TGF-treated) counterparts by looking at the EVs they produced. Looking at our dataset, there were 78 proteins persistently found in ROL/PA and S80 groups that were absent in the persistent protein profile of EVs originating from TGF-treated cells. Conversely, there were 4 proteins in the TGF group that were not consistently found in ROL/PA and S80 (Fig. 3f). The thus selected proteins from our data were looked up consulting the UniProt database ${ }^{48}$ in an effort to narrow down the lists to find candidate protein markers within these two subsets that were either tissue-specific, membrane-bound and/or secreted. Tissue specificity would be desirable for the translational applicability of our protocols, opening up the possibility of analyzing more complex ex vivo samples of EVs, while possibly being able to trace the EVs back to their origin $^{49}$. We hypothesized that proteins that have been reported to be membrane-bound and/or secreted are less likely to be in the inner core of EVs. If so, they could be detected on the surface of EVs without destroying them. We have thus rationally identified reduced lists of possible protein markers for our purposes from the ROL/PA and S80 subset (22 candidates), and from the TGF subset (4 candidate proteins). 
a

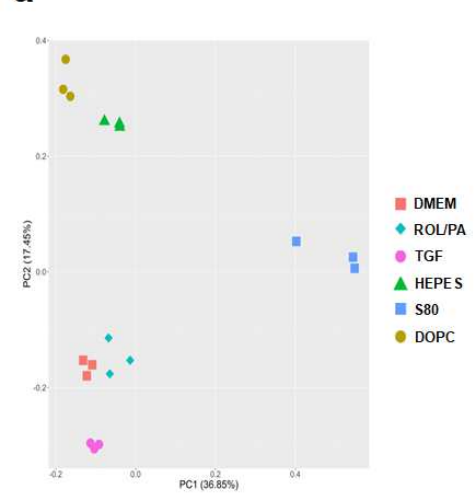

C

\begin{tabular}{cccc}
\hline & $\begin{array}{c}\text { Persistent } \\
\text { proteins }\end{array}$ & $\begin{array}{c}\text { Persistent proteins } \\
\text { in addition to ALL }\end{array}$ & $\begin{array}{c}\text { Exclusive } \\
\text { proteins }\end{array}$ \\
\hline AL SAMPLES & 1931 & - & - \\
DMEM & 2555 & 624 & 48 \\
ROUPA & 2564 & 633 & 50 \\
TGF & 2388 & 457 & 28 \\
HEPES & 2674 & 743 & 46 \\
S 80 & 3163 & 1232 & 479 \\
DOPC & 2451 & 520 & 53
\end{tabular}

d b
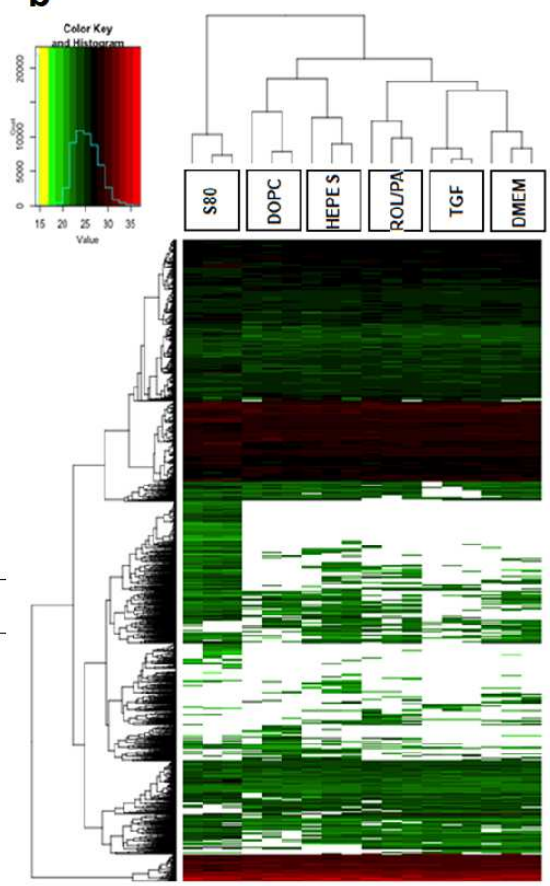

DMEM ROL/PA TGF DOPC HEPES $\$ 80$

\begin{tabular}{ll|l|l|l|l|l|l|l}
0.131041 & 0.143962 & 0.097731 & 0.049667 & 0.219253 & 0.358346 & EXOC5
\end{tabular} $\begin{array}{lllllll}0.23759 & 0.184593 & 0.177327 & 0.194043 & 0.103549 & 0.102899 & \text { CCS }\end{array}$ $\begin{array}{llllllllll}0.082487 & 0.119659 & 0.121999 & 0.070899 & 0.068961 & 0.535995 & \text { SNUT1 }\end{array}$ \begin{tabular}{lllllllll|l}
0.046227 & 0.047696 & 0.048711 & 0.032097 & 0.05194 & 0.773329 & TGF11
\end{tabular}

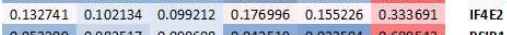

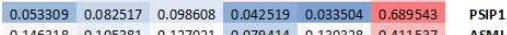

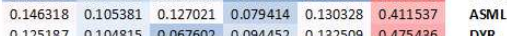
$\begin{array}{lllllllll}0.125187 & 0.104815 & 0.067602 & 0.094452 & 0.132509 & 0.475436 & \text { DYR }\end{array}$ \begin{tabular}{lll|l|l|l|l|l|l|l|l}
0.149243 & 0.118704 & 0.487867 & 0.101843 & 0.033909 & 0.108434 & CO1A
\end{tabular}

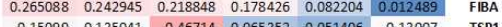
\begin{tabular}{rrrrrrrr}
0.15099 & 0.135041 & 0.46714 & 0.065352 & 0.051406 & 0.13007 & TSP1 \\
\hline 0.055054 & 0.087459 & 0.063442 & 0.045598 & 0.038399 & 0.710047 & TOP
\end{tabular} $\begin{array}{lllllllll}0.055054 & 0.087459 & 0.063442 & 0.045598 & 0.038399 & 0.710047 & \text { TOP1 }\end{array}$ $\begin{array}{llllllllll}0.146998 & 0.106521 & 0.649987 & 0.047058 & 0.040746 & 0.00869 & \text { CSPG2 }\end{array}$ \begin{tabular}{llllllllllll}
0.114099 & 0.141511 & 0.067861 & 0.159889 & 0.19972 & 0.316921 & NQO1 \\
\hline & 0.117776 & 0.11104 & 0.061021 & 0.19661 & 0.188027 & 0.325526 & RFA2
\end{tabular}

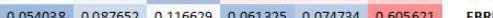
$\begin{array}{llllllllll}0.054038 & 0.087052 & 0.110629 & 0.001325 & 0.074734 & 0.005021 & \text { FBRL }\end{array}$ \begin{tabular}{ll|lllllllll}
0.213238 & 0.182828 & 0.103304 & 0.240875 & 0.149222 & 0.11053 & ENPP1 \\
\hline
\end{tabular} $\begin{array}{llllllllll}0.080853 & 0.127724 & 0.047015 & 0.035018 & 0.092004 & 0.517377 & \text { HNRH2 }\end{array}$

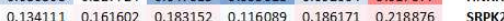
$\begin{array}{lllllllllll}0.126516 & 0.11533 & 0.092473 & 0.089154 & 0.087846 & 0.488681 & \text { CDK17 }\end{array}$

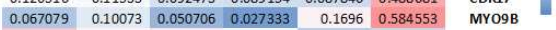
$\begin{array}{lllllllll}0.354338 & 0.158485 & 0.211064 & 0.117706 & 0.07429 & 0.084116 & \text { ITSN1 }\end{array}$

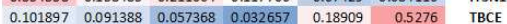
\begin{tabular}{|l|l|l|l|l|l|l|l|}
0.1111775 & 0.152392 & 0.181405 & 0.107127 & 0.120196 & 0.327104 & SRSF7
\end{tabular}

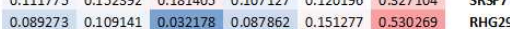
\begin{tabular}{l|l|l|l|l|l|l|l|l|l}
0.096081 & 0.189733 & 0.03494 & 0.048668 & 0.165154 & 0.465423 & RHG29 & EF1
\end{tabular} \begin{tabular}{lllllllll|l}
0.187691 & 0.270953 & 0.354989 & 0.098817 & 0.064021 & 0.023529 & MUC1
\end{tabular}

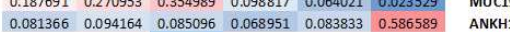
$\begin{array}{lllllllll}0.132783 & 0.163698 & 0.038042 & 0.121133 & 0.158974 & 0.38537 & \text { EH11 }\end{array}$

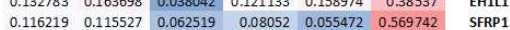
$\begin{array}{lllllllllll}0.130536 & 0.140481 & 0.080074 & 0.173069 & 0.177018 & 0.298822 & \text { CPSF7 }\end{array}$ \begin{tabular}{|l|l|l|l|l|l|l|l|l}
0.062216 & 0.068583 & 0.066066 & 0.079212 & 0.130432 & 0.593492 & FA98A
\end{tabular} $\begin{array}{lllllllll}0.035262 & 0.086115 & 0.060899 & 0.043234 & 0.056064 & 0.718427 & \text { PRP31 }\end{array}$ $\begin{array}{lllllllll}0.190427 & 0.246698 & 0.18235 & 0.101399 & 0.190035 & 0.089091 & \text { VMP1 }\end{array}$ $\begin{array}{llllllllll}0.078538 & 0.085823 & 0.071195 & 0.072456 & 0.081349 & 0.610638 & \text { QKI }\end{array}$ $\begin{array}{lllllllll}0.178892 & 0.241125 & 0.089596 & 0.071678 & 0.132973 & 0.285736 & \text { UACA }\end{array}$ $\begin{array}{llllllll}0.135858 & 0.118225 & 0.077794 & 0.257036 & 0.197343 & 0.213743 & \text { PNPO }\end{array}$ $\begin{array}{lllllllll}0.143909 & 0.190258 & 0.081586 & 0.1846 & 0.203068 & 0.196579 & \text { ARP10 }\end{array}$ \begin{tabular}{lllllll|l}
0.146503 & 0.190384 & 0.174792 & 0.07894 & 0.126025 & 0.283356 & OGFR
\end{tabular} $\begin{array}{lllllll}0.106136 & 0.123526 & 0.12567 & 0.059337 & 0.139446 & 0.445885 & \text { ABCF2 }\end{array}$

\begin{tabular}{c|cccccc}
$\begin{array}{c}\text { [shared proteins } \\
\text { (exclusively shared)] }\end{array}$ & DMEM & ROLIPA & TGF & HEPES & S80 & DOPC \\
\cline { 2 - 6 } DMEM & - & $383(13)$ & $313(15)$ & $394(11)$ & $395(22)$ & $282(10)$ \\
ROLIPA & & - & $297(10)$ & $388(16)$ & $416(35)$ & $250(2)$ \\
TGF & & & - & $266(3)$ & $269(17)$ & $171(3)$ \\
HEPES & & & & - & $514(82)$ & $357(31)$ \\
S80 & & & & & - & $320(16)$ \\
DOPC & & & & & & -
\end{tabular}

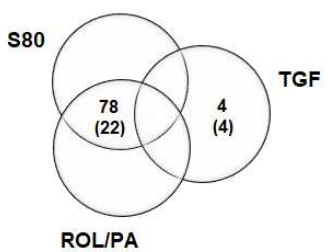

Fig. 3 | Main proteomic findings from SEC purified EVs originating from differently treated LX-2. a,b Principal component analysis and hierarchical clustering showing similarity degrees between biologically independent samples undergoing the same treatments, and differences between treatment groups. c, Summary of the number of persistent proteins found in each treatment group. d, Summary of the number of persistent proteins shared across treatment groups, excluding the 1'931 shared by all. e, Panel resulting from cross-referencing the proteins consistently found in all treatment groups and the proteins that were under or over expressed in a least one direct comparison after Welch's t-test. Expression levels were scored by normalizing to the sum of LFQ intensities to generate a heat map. f, Venn diagram depicting the number of identified proteins persistently found in S80 and ROL/PA groups as opposed to TGF and vice versa; numbers in parenthesis indicate proteins within those subsets which were either tissue specific, membranebound and/or secreted. See SI file 2021_czivko_r01_proteins. 


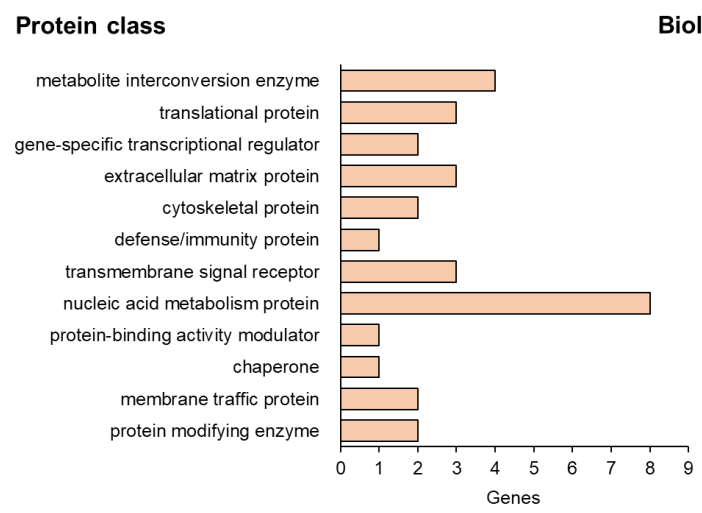

Biological process
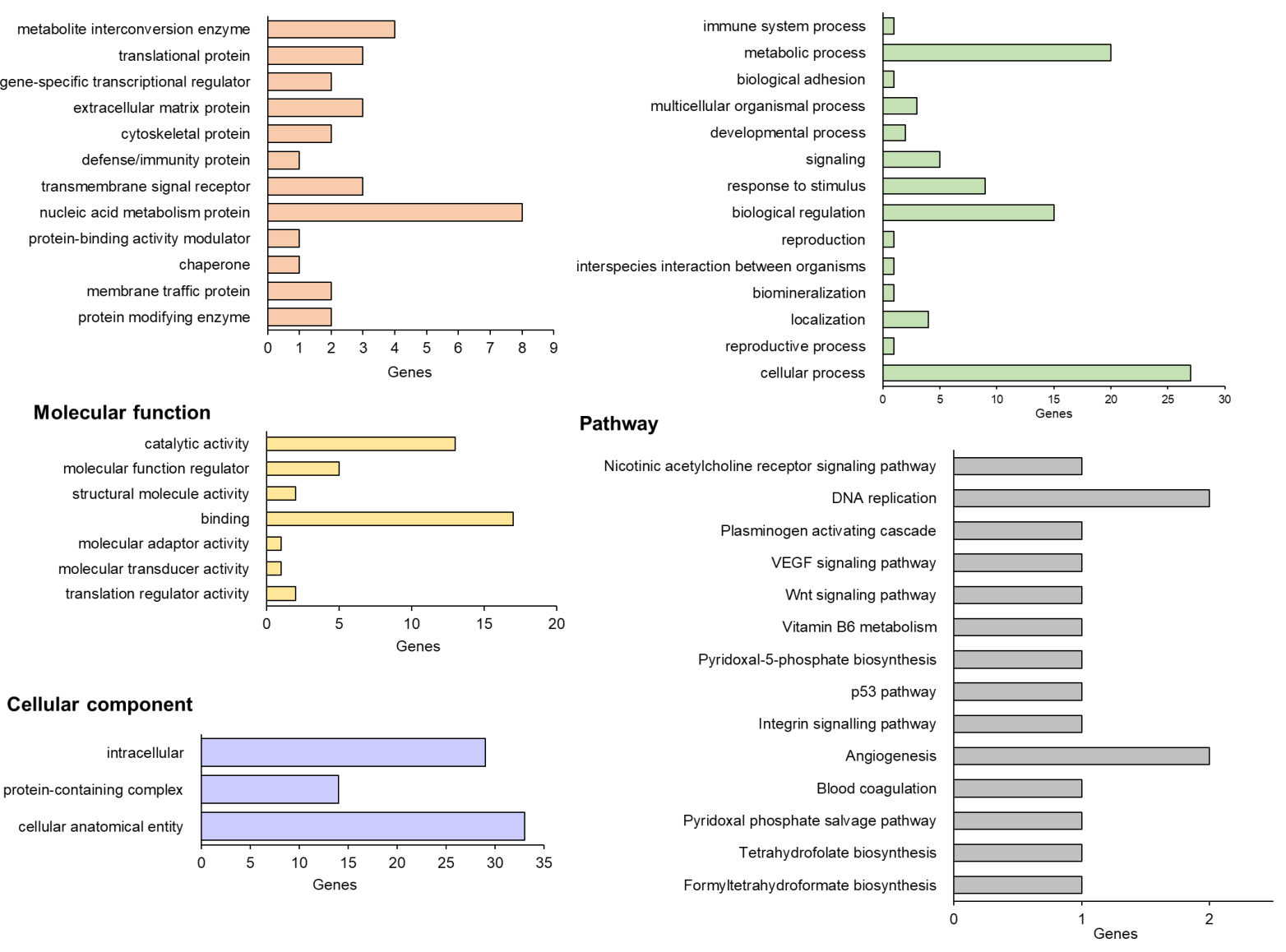

Fig. 4 | Representative Classification of the 44 proteins comprising the treatment-discriminating panel using the PANTHER platform.

\section{Proteomic analysis of AF4-purified EVs.}

AF4 is a powerful technique increasingly adopted for the purification of nano-sized particles, polymers, protein complexes, viruses, and even EVs ${ }^{50-53}$. During AF4 purification of our EV samples, two main peaks emerged, and we collected both the early and late eluting peak (peak 1 and peak 2, respectively) (Fig. 1). Similar to the proteomic analysis performed for the SEC-purified EVs, AF4-purified samples were examined with the purpose of finding lists of treatment-discriminating proteins. We investigated peak 1 and peak 2 separately, as well as combined, leading to the identification of a total of 1,807 distinct proteins. Compared to the SEC-purified EV protein profiles, AF4-purified EVs produced shorter lists. The explanation can be twofold. First, the SEC EV-containing peak was just one, and sharper than either of the collected AF4-peaks. This directly affects the precision with which the EV-associated proteins can be isolated, especially when considering that some could be found in both or either one of the AF4-peaks. Secondly, AF4-fractions are more diluted and limited in terms of recovered EVs, and thus in the recovered EVassociated proteins. 
PCA revealed that peak 2 resulted in better grouping of treatments compared to peak 1 . This better grouping indicates that this particular fraction holds a distinctive EV-subset that could be used for treatmentdiscrimination (Figs. 5a,b,c). Nonetheless, combined analysis of AF4 peaks showed a substantial number of proteins that can be found across both bands, resulting in mixed hierarchical clustering even when looking at peak 2 alone (Fig. 5d), and even after imputation (Fig. S10).

As with SEC-purified EVs, lists of treatment-correlating, persistent proteins were generated for AF4-purifed EVs, both by looking at the peaks separately and by looking at the peaks combined (Figs. 5e,f). Every treatment group was compared to each of the other ones: volcano plots for all the single comparisons are found in Figs. S11-13. The generated lists also allowed for a comparison of SEC and AF4 purification methods, summarized with Venn diagrams (Fig. $5 \mathrm{~g}$ ). The vast majority of the proteins found by AF4 purification were also detected in SEC-samples. AF4-peak 2 had more original hits compared to AF4-peak 1 , and indeed proteins isolated from AF4-peak 2 have a greater overlap with SEC-findings. Purification by AF4 led to the confirmation of many of the proteomic findings from SEC-purified EVs, split across two distinctive subpopulations of EVs, which would warrant further exploration in the future. 

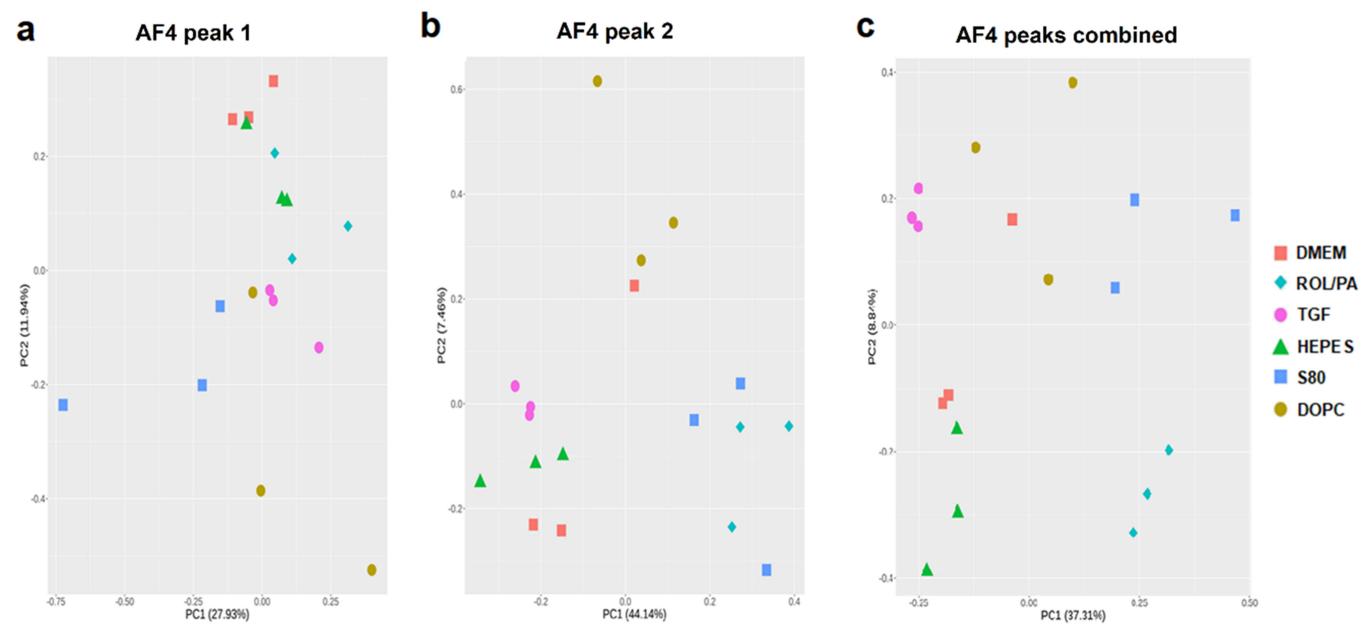

d

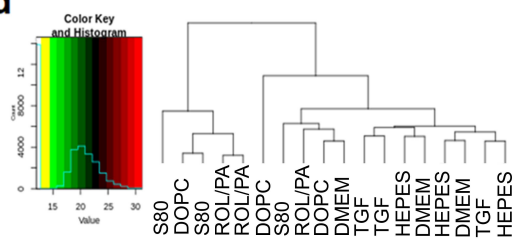

e

\begin{tabular}{cccc}
\hline & $\begin{array}{c}\text { Persistent proteins } \\
\text { AF4 peak 1 } \\
\text { (group exclusives) }\end{array}$ & $\begin{array}{c}\text { Persistent proteins } \\
\text { AF4 peak 2 } \\
\text { (group exclusives) }\end{array}$ & $\begin{array}{c}\text { Persistent proteins } \\
\text { AF4 peaks combined } \\
\text { (group exclusives) }\end{array}$ \\
\hline ALL & 69 & 348 & 407 \\
DMEM & $472(60)$ & $551(5)$ & $689(14)$ \\
ROL/PA & $254(2)$ & $1043(283)$ & $1078(177)$ \\
TGF & $363(16)$ & $531(4)$ & $638(6)$ \\
HEPES & $378(16)$ & $504(2)$ & $639(3)$ \\
S80 & $395(34)$ & $858(33)$ & $946(70)$ \\
DOPC & $97(3)$ & $495(3)$ & $525(3)$
\end{tabular}

f

\begin{tabular}{|c|c|c|c|c|c|c|}
\hline $\begin{array}{l}\text { shared proteins } \\
\text { (shared exclusively) }\end{array}$ & DMEM & ROL/PA & TGF & HEPES & $\mathbf{S} 80$ & DOPC \\
\hline DMEM & _ & $232(14)$ & $154(3)$ & $164(2)$ & $215(15)$ & $59(0)$ \\
\hline ROL/PA & & _ & $198(4)$ & $211(4)$ & $429(143)$ & $107(4)$ \\
\hline TGF & & & _ & 139 (1) & $186(5)$ & $40(1)$ \\
\hline HEPES & & & & _ & $189(3)$ & $52(0)$ \\
\hline$S 80$ & & & & & _ & $98(2)$ \\
\hline DOPC & & & & & & - \\
\hline
\end{tabular}

g
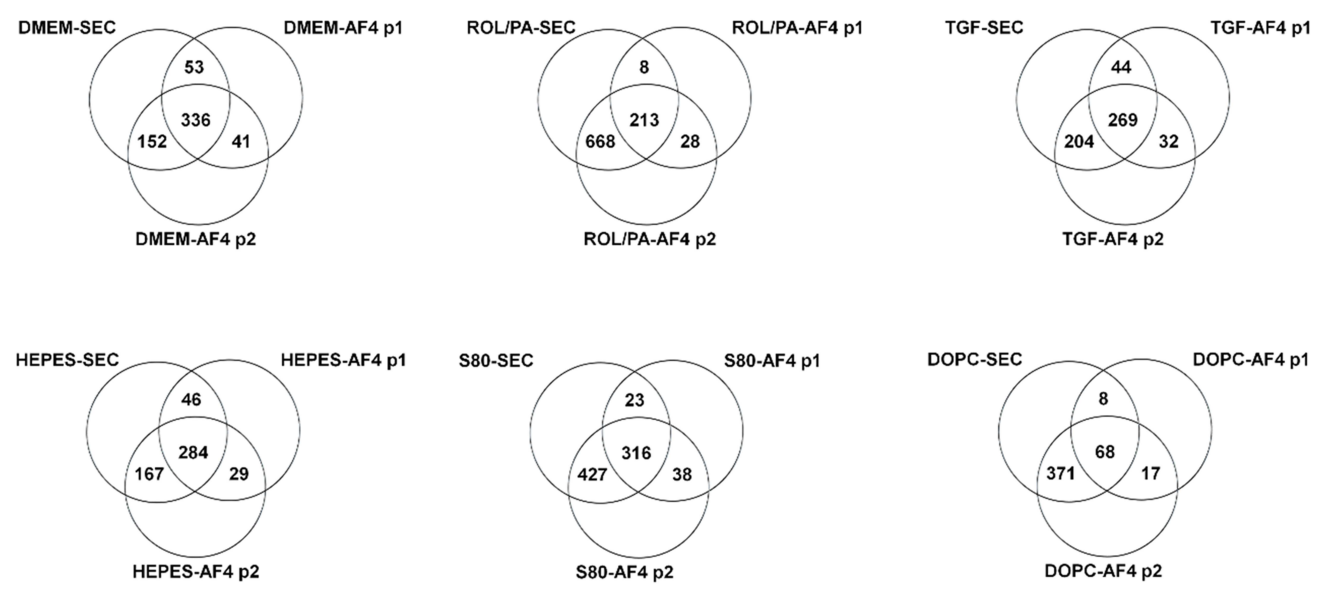
Fig. 5 | Main proteomic findings from AF4 purified EVs originating from differently treated LX-2. a,b,c Principal component analysis for samples originating from AF4 peak 1 (a), peak 2 (b) and for the two peaks combined (c). d, Hierarchical clustering for biologically independent AF4-peak 2 samples undergoing the same treatments. e, Summary of the number of persistent proteins found in each treatment group. $\mathbf{f}$, Summary of the number of persistent proteins shared across treatment groups. g, Venn diagram comparisons of SEC and AF4 purified samples: for every treatment group, the proteins found in the SEC fraction are compared to those found in AF4-peak1 (AF4 p1) and AF4-peak2 (AF4 p2).

\section{Fluorescence NTA method for the detection of EV-associated proteins correlating to cell status.}

The detection of specific proteins on EVs is an integral part of the field. Typically, western blot is the method of choice to show the presence of known exosomal markers in EV samples, including CD9, CD81, CD63, and Alix. ${ }^{54-60}$ Alternatively, strategies relying on flow cytometry (FACS) are employed. Using FACS when working with EVs can be challenging, given that the instruments were designed for the analysis of single cells, which are considerably larger in size ${ }^{61}$. A workaround for this particular issue is the coupling of EVs to large beads, and subsequently analyzing the presence of the markers of interest on the beads covered in EVs. Nano-flow cytometers have recently entered the market for the specific analysis of nanoparticles in a way that is analogous to how classical FACS instruments work with full cells ${ }^{62-65}$. Research groups have been trying to measure fluorescently labeled EVs directly by fluorescence nanoparticle tracking analysis (f-NTA), demonstrating the feasibility of its application ${ }^{58,66,67}$. These reports used unspecific dyes or immunolabeling for specific proteins, often opting for quantum dots (QD) conjugated antibodies to overcome photostability problems associated with many conventional fluorophores ${ }^{58}$. The latter approach, however, can be vitiated if effective protocols to purify QD-labelled EVs from free QD are not effectively validated.

Our proteomic analysis of EVs has yielded a list of candidate protein markers which could be detected on the surface of EVs without destroying them, thus allowing for their analysis by f-NTA. SPARC ${ }^{31,32,42,68}$ was thus selected as a proof-of-concept protein, since it was one of the 4 proteins consistently found in TGF samples, but not in ROL/PA and S80 (Fig. 4f). We demonstrated that, while not necessarily an integrated part of EV-membranes, SPARC was associated strongly enough with EVs to be co-purified after SEC and AF4. SPARC is also reportedly secreted or found in the extracellular region, in or around the basement membrane, indicating that when found in EV samples, it is more likely to be associated with their membrane than with their aqueous inner compartment. Importantly, SPARC can be highly expressed in tissues undergoing wound repair or morphogenesis ${ }^{31,69,70}$, including the liver ${ }^{71,72}$, making it overall an excellent candidate for the reporting on the physiological state of the cells that released the EVs with which SPARC could be found ${ }^{73,74}$.

To develop immunolabeling methods for the non-destructive analysis of physiologically relevant EVassociated proteins by f-NTA, we first assessed the feasibility of detecting fluorescently labeled EVs in general. $\mathrm{PKH}^{\circledR}$ dyes are used in a wide variety of instances for the non-specific labelling of cellular and vesicular membranes ${ }^{75-77}$. SEC-purified EVs from untreated LX-2 cells were successfully labelled with the PKH67 membrane dye ${ }^{78}$. Testing different concentrations over time, we could determine that the labeling plateaued after $15 \mathrm{~min}$ (Fig. S14). We settled on $20 \mathrm{~min}$ of incubation time when we systematically 
increased dye concentration, showing that almost all detected particles were indeed membranous, and that an almost linear dose-dependency could be reliably measured $\left(R^{2}=0.909\right.$, Fig. $\left.6 a\right)$. The use of PKH dyes has been recently called into question, particularly because of their hydrophobicity leading to the formation of dye aggregates that can be detected by NTA, significantly affecting the size distribution profile ${ }^{79,80}$. However, given the freshly prepared PKH67 concentrations that we used in our samples with relatively few EVs (Table S1), we did not encounter new subpopulations of nanoparticles in our measurements compared to unstained samples (for representative size distribution profiles of PKH67 labeled particles see Fig. S15). The next steps involved labelling EVs with an AlexaFluor488-conjugated secondary antibody. We chose to start with CD81, since we found it to be present in all EV samples in our proteomic analyses. Being a tetraspanin, it was also likely to be available for binding without destroying the EVs, and previous reports have shown that it could be detected by $\mathrm{f}-\mathrm{NTA} \mathrm{A}^{58,66}$.

A first approach was to incubate AF488-CD81 directly in the SEC-purified EV samples originating from DMEM-treated LX-2 cells, at different dilutions of AF488-CD81, for various amounts of time and at different temperatures. Higher concentrations increased the number of detected particles in fluorescence mode, but not in a dose-dependent manner (Fig. 6b). Incubation at $37^{\circ} \mathrm{C}$ was not significantly better than incubation at room temperature, although fluorescent particles were detectable starting from an earlier time point (Fig. $\mathrm{S16}$ ). Incubation time consistently had a greater influence, and we found the range between 4 and $6 \mathrm{~h}$ optimal for our samples. Longer times $(18 \mathrm{~h})$ could be tested at lower temperatures $\left(4^{\circ} \mathrm{C}\right)$ to split the workload of EV-isolation and EV-analysis, while minimizing EV-loss.

Without a purification step for excess AF488-CD81 prior to measurement, the background intensity was always too high for the software, regardless of final dye dilution. Our relatively low EV-yields resulted in limited final dilutions of the SEC fractions before NTA measurements. More concentrated EV samples could be labeled after SEC with fewer issues.

To obviate these limitations, we decided to incubate AF488-CD81 with the EV-pellet resuspended after UC, right before the SEC purification we performed regardless. The biggest drawback of this incubation strategy is the limited number of EV-pellets that can be obtained in one day. Additionally, it slows down the already time-limiting SEC step. There was no significant difference between 3 and $5 \mathrm{~h}$ incubation times, other than the smaller standard deviations for the latter instance, which is why we chose it for further experiments (Fig. $6 c)$.

Since $8 \mathrm{ng} / \mathrm{mL}$ resulted in higher labelling, that concentration was used when we looked at CD81 and CD9 labeling both separately and combined (Fig. 6d). While we can neither confirm nor exclude co-localization of the two markers on a single EV, the combined results suggest that there is some incomplete overlap. This means that double or even triple staining with exosomal markers might be a viable strategy to cover $100 \%$ of the particles detected in scatter mode for a sample of pure exomes. Antibody concentrations for incubations with EV-pellets could be further optimized to account for EV-abundance in the samples.

As a final step, we compared our newly developed f-NTA methods to the analysis of EV-markers by FACS using $6 \mathrm{ng} / \mathrm{mL}$ of AF488-CD9 (Fig. 6e). Both methods detected CD9 positive events in all samples- 
irrespective of cell treatment prior to EV harvest - which validated the presence of exosomal markers in our EV populations by all three proteomics, FACS and f-NTA. FACS analysis resulted in higher percentages of fluorescently labelled events (up to 70\%) compared to f-NTA (around $30 \%$ ), but also with considerably higher standard deviations. Our f-NTA methods on the other hand performed more reliably, especially considering that there were no false positives, i.e., no particles could be detected in samples incubated with isotype controls.

Our optimized immunolabeling methods for the detection of proteins on single EVs by f-NTA could be transferred to check for the presence of SPARC in EVs isolated from differently treated LX-2 cells. f-NTA measurements were consistent with proteomic findings. The presence of CD81 was consistent across samples, and the level of SPARC associated with EVs was reproducibly different for different treatment groups, with HEPES and TGF samples having the highest amounts, and S80 especially having hardly any (Fig. 6f). We found that $S 80$ greatly reduces the relative presence of SPARC-positive EVs. We are currently investigating whether S80 actively suppress SPARC, or whether S80's mechanism of action results in lower SPARC expression. SPARC presence in EV-samples from DOPC-treated cells (less than 10\%) was also decidedly lower than for HEPES $(70 \%)$ and TGF $(40 \%)$ in our f-NTA measurements.

We do not know by which mechanism SPARC is being associated with EVs. The higher presence in some of our samples compared to others could be directly linked to an increased expression of the protein. It could also be due to an improved affinity to EV-surface, either because of treatment-induced changes in EV-composition (see Fig. 3) or because of treatment-induced differences in the extracellular chemical environment, which might lead to a worsening of SPARC's affinity to the ECM, for example.

Nevertheless, these results prove that f-NTA could be used for a quantitatively meaningful detection of physiologically relevant EV-associated proteins by incorporating the incubation of secondary antibodies into rigorously established EV isolation and purification steps. This ultimately provides a convenient alternative to more time-consuming and hands-on heavy western blot and flow cytometry protocols. Moreover, for the first time, we could measurably correlate the cellular response to PPC treatments such as $\mathrm{S} 80$ to the lowering of the relative presence of SPARC in EVs generated by HSCs, offering novel insights into the (therapeutic) effect of PCC treatments. 

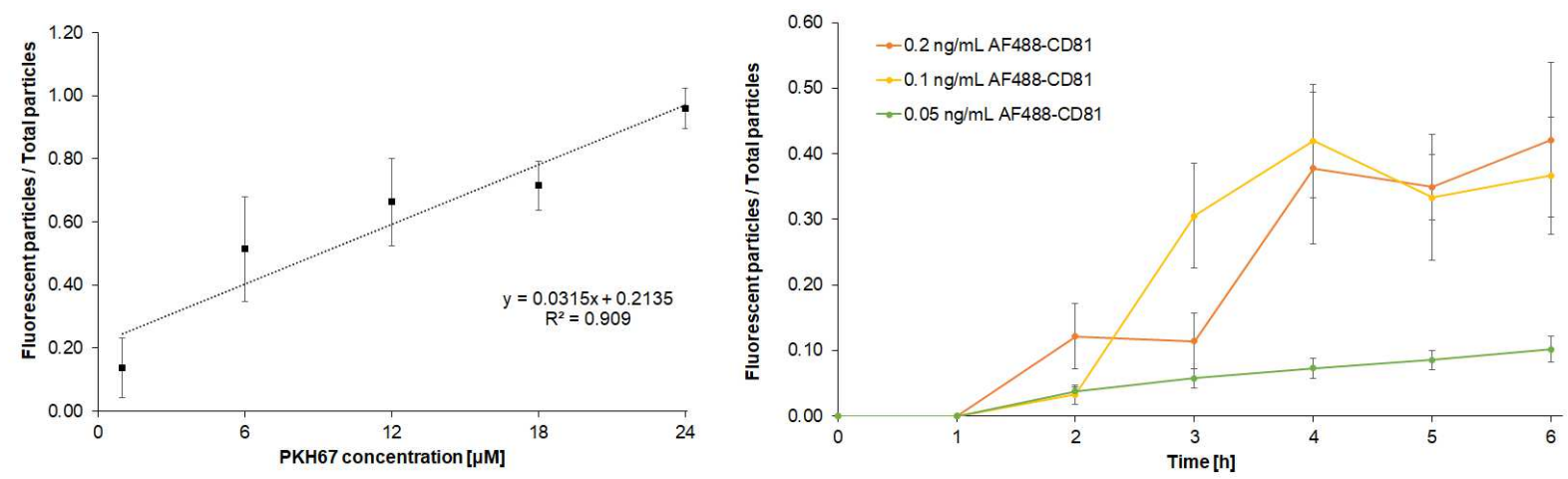
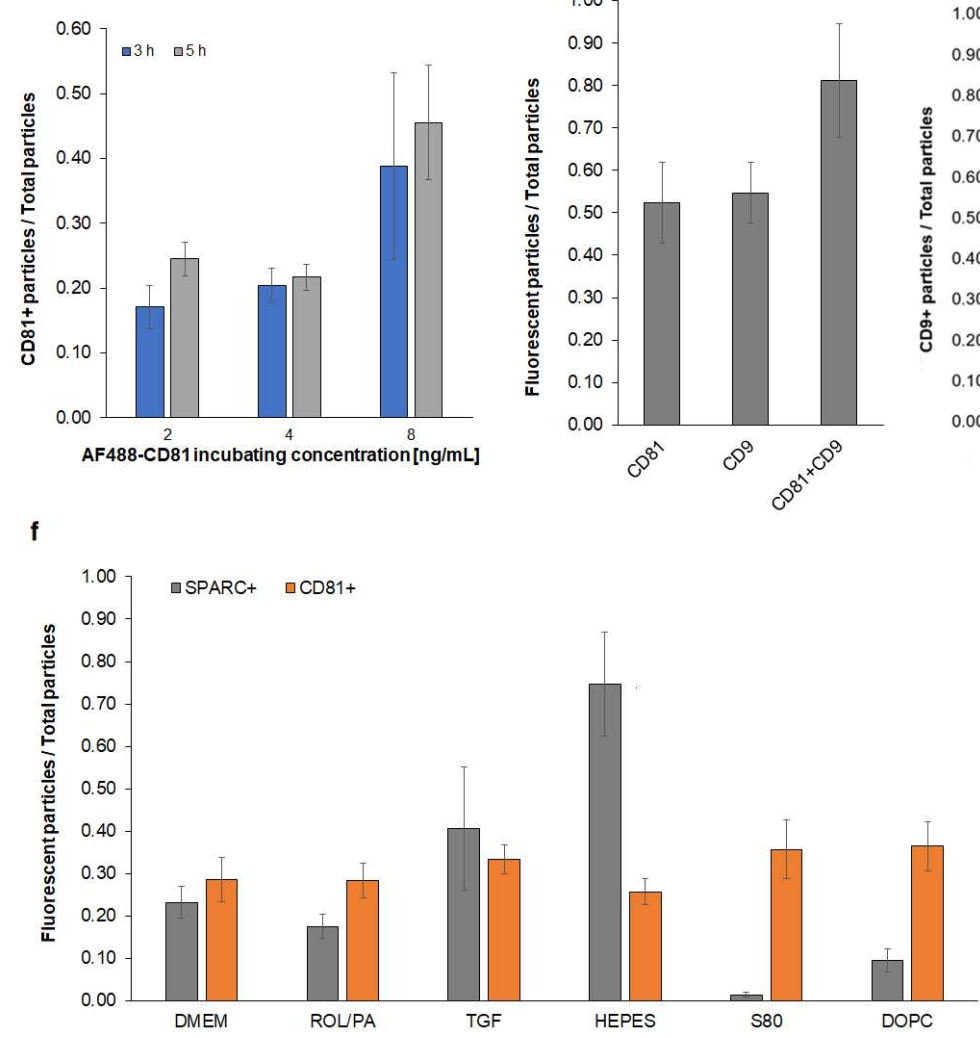

e
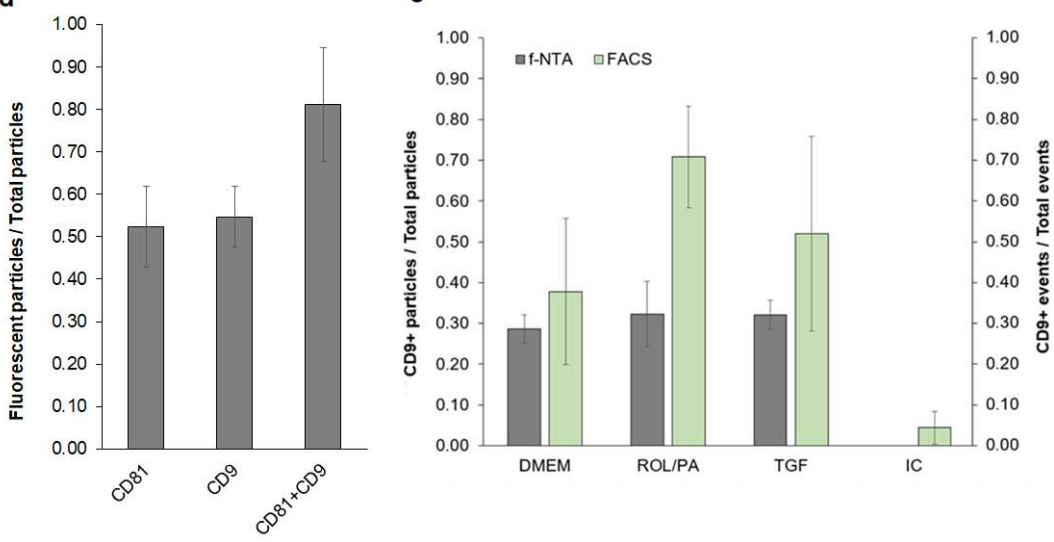

Fig. 6 | Feasibility of f-NTA for the detection of physiologically relevant proteins. a, SEC-purified EVs stained with unspecific membrane dye PKH67 by systematically increasing dye concentrations. b, SECpurified EVs incubation with varying amounts of AF488-CD81 and for different times at $24{ }^{\circ} \mathrm{C}$. c,d EVcontaining pellets incubated with AF488-CD81 prior to SEC and detected after purification (c) and comparison with additional incubation with AF488-CD9 (d). e, Direct comparison of CD9 detection by f-NTA and FACS using EVs from differently treated LX-2, including isotype control (IC). f, Detection of SPARC and CD81 on EVs isolated from differently treated LX-2 cells following the optimized f-NTA protocol (mean $\pm S D, n=3)$. 


\section{Discussion}

We reported on highly reproducible isolation and purification methods for EVs released by LX-2 HSCs upon six different treatments, affecting the LX-2 phenotype in different ways and to different extents. The heterogeneity of EV samples has hindered the research into distinctive subpopulations of EVs due to their overlapping sizes, densities, zeta potential values and biochemical compositions ${ }^{39}$. We were nonetheless able to observe subtle size shifts within these polydisperse samples of EVs originating from differently treated cells by NTA, as well as prominent dissimilarities in EAF4. The groundwork EV-analysis optimized herein begs for further methods of characterization such as the inspection of RNA or glycosylation patterns. Nonetheless, our EV-analysis for an immortalized cell line used as widely as LX-2 is pivotal to boost more investigations into possible antifibrotic treatments by evaluating the resulting EVs.

When used to treat fresh cells, EV-pellets from differently treated cells were sufficient to induce a physiological response similar to direct treatment, as observed by fluorescence microscopy. However, the investigation into the biological role of EVs is also far from being exhausted. It would be important to see for how long after treatment do HSCs preserve the phenotypical changes acquired. Importantly, we do not know for how long would their EVs be effective in causing those same phenotypical changes onto naïve cells, and how do the protein profiles of the latter's EVs look like.

Proteomic data of SEC and AF4-purified EVs was mined to find candidate protein markers to discriminate EV samples between differently treated HSCs. Although beyond the scope of this particular study, our proteomic data can be further examined by detailed gene set enrichment analysis (e.g., network and pathway analyses) for all the generated protein lists. We summarized more than 120 distinctive lists of proteins in our tables. Deep learning approaches could be used to sort through different EV-proteomic data to find the minimum, specific signature profile for HSCs, similarly to what had been recently done for the identification of EVs originating from cancer cells ${ }^{60}$.

SPARC was rationally selected to successfully explore the feasibility of using f-NTA as a non-destructive tool for the aforementioned discriminatory exercise. By thus looking into the relative presence of SPARCpositive EVs, we found that the PPC-rich phospholipid S80 greatly reduces it. In the future, our optimized f-NTA method could be applied to EVs from LX-2 treated differently, allowing screening drug candidates targeting fibrogenesis. While SPARC proved to be an excellent marker for negative impact of S80 on HSCs' fibrotic phenotype in our model, having a working candidate protein to report on positive phenotypes would be all the more meaningful, especially with a side-by-side comparison. We have tried to select proteins from the quiescent-like status list (Fig. 3f) which could be detected non-destructively, namely GPC1 and IKKB, but we were unable to detect effective immunolabeling with either one of them by f-NTA. However, there are at least almost 20 more interesting proteins to test from our data. The method could be expended to include more EV-associated, clinically relevant proteins, even within the same sample. NTA systems with more than one laser and fluorescence channel would allow for the almost simultaneous analysis of multiple proteins, provided they are labelled with non-interfering dyes. While co-localization of single proteins onto 
single EVs would not be directly measurable by NTA yet, their collective presence within the same EV samples is already technologically possible to verify.

Our results pave the way for more precise clinical analyses: markers related to diseased and healthy states, as well as proteins that are tissue specific or preferentially expressed by specific cells could all be conceivably checked within one EV sample if appropriately selected. Assessing the translational applicability of our protocols for the evaluation of EVs from primary cells, or blood from healthy volunteers or patients would be the most important prospective validation to accomplish.

\section{Acknowledgements}

The Phospholipid Research Center is kindly acknowledged for the financial support (Grant ID: PLU-2017056/2-1). C.Z. and P.L. would like to thank the staff of the Proteomics and Mass Spectrometry Core Facility (PMSCF), Department for BioMedical Research (DBMR), University of Bern, Switzerland. The morphology of the isolated EVs was evaluated under scanning electron microscopy (SEM) with the help of Dr. Chiara De Rossi (Helmholtz Institute for Pharmaceutical Research, Saarbrücken). Cryogenic transmission electron microscopy (cryo-TEM) imaging was performed with the help of Dr. Jana Thamm (Friedrich Schiller University, Jena). G.F. would like to acknowledge financial support from the NanoMatFutur program from the Federal Ministry of Research and Education (grant number 13XP5029A).

\section{Authors' contribution statements}

P.L. and G.F. conceived the original project and supervised it. C.Z. devised and performed all the experiments and analyzed all data. K.F. devised, carried out and analyzed the EAF4 experiments. C.Z. led manuscript writing. P.L. and G.F. interpreted data and revised the manuscript. All authors contributed to discussion.

\section{References}

1. Trefts, E., Gannon, M. \& Wasserman, D. H. The liver. Curr. Biol. 27, R1147-R1151 (2017).

2. Zivko, C., Fuhrmann, G. \& Luciani, P. Liver-derived extracellular vesicles: A cell by cell overview to isolation and characterization practices. Biochim. Biophys. Acta - Gen. Subj. 1865, 129559 (2021).

3. Asrani, S. K., Devarbhavi, H., Eaton, J. \& Kamath, P. S. Burden of liver diseases in the world. J. Hepatol. 70, 151-171 (2019).

4. Byass, P. The global burden of liver disease: A challenge for methods and for public health. BMC Medicine 12, (2014).

5. Pimpin, L. et al. Burden of liver disease in Europe: Epidemiology and analysis of risk factors to identify prevention policies. J. Hepatol. 69, 718-735 (2018). 
6. Marcellin, P. \& Kutala, B. K. Liver diseases: A major, neglected global public health problem requiring urgent actions and large-scale screening. Liver Int. 38, 2-6 (2018).

7. Ndugga, N. et al. Disparities between research attention and burden in liver diseases: Implications on uneven advances in pharmacological therapies in Europe and the USA. BMJ Open 7, (2017).

8. Tsuchida, T. \& Friedman, S. L. Mechanisms of hepatic stellate cell activation. Nat. Rev. Gastroenterol. Hepatol. 14, 397-411 (2017).

9. Gandhi, C. R. Hepatic stellate cell activation and pro-fibrogenic signals. J. Hepatol. 67, 1104-1105 (2017).

10. Bataller, R. \& Brenner, D. A. Hepatic stellate cells as a target for the treatment of liver fibrosis. Semin. Liver Dis. 21, 437-451 (2001).

11. Parola, M. \& Pinzani, M. Liver fibrosis: Pathophysiology, pathogenetic targets and clinical issues. Mol. Asp. Med. 65, 37-55 (2019).

12. Sircana, A., Paschetta, E., Saba, F., Molinaro, F. \& Musso, G. Recent Insight into the Role of Fibrosis in Nonalcoholic Steatohepatitis-Related Hepatocellular Carcinoma. Int. J. Mol. Sci. 20, 1745 (2019).

13. Cheng, J. Y.-K. \& Wong, G. L.-H. Advances in the diagnosis and treatment of liver fibrosis. Hepatoma Res. 3, 156 (2017).

14. Singh, S., Venkatesh, S. K., Wang, Z. \& al., et. Diagnostic performance of magnetic resonance elastography in staging liver fibrosis: a systematic review and meta-analysis of individual participant data. Clin. Gastroenterol. Hepatol. 13, 440-451.e6 (2015).

15. Pavlov, C. S., Casazza, G., Nikolova, D. \& al., et. Systematic review with meta-analysis: diagnostic accuracy of transient elastography for staging of fibrosis in people with alcoholic liver disease. Aliment Pharmacol Ther 43, 575-585 (2016).

16. Boursier, J. et al. New sequential combinations of non-invasive fibrosis tests provide an accurate diagnosis of advanced fibrosis in NAFLD. J. Hepatol. 71, 389-396 (2019).

17. Friedrich-Rust, M., Ong, M. F., Martens, S. \& al., et. Performance of transient elastography for the staging of liver fibrosis: a meta-analysis. Gastroenterology 134, 960-974 (2008).

18. Friedman, S. L. Mechanisms of Hepatic Fibrogenesis. Gastroenterology 134, 1655-1669 (2008).

19. Pellicoro, A., Ramachandran, P., Iredale, J. P. \& Fallowfield, J. A. Liver fibrosis and repair: Immune regulation of wound healing in a solid organ. Nat. Rev. Immunol. 14, 181-194 (2014).

20. Fuhrmann, G., Herrmann, I. K. \& Stevens, M. M. Cell-derived vesicles for drug therapy and diagnostics: Opportunities and challenges. Nano Today 10, 397-409 (2015).

21. Sato, K. et al. Intercellular communication between hepatic cells in liver diseases. Int. J. Mol. Sci. 20, 2180 (2019).

22. Van Niel, G., D’Angelo, G. \& Raposo, G. Shedding light on the cell biology of extracellular vesicles. Nat. Rev. Mol. Cell Biol. 19, 213-228 (2018).

23. van der Pol, E., Böing, A. N., Harrison, P., Sturk, A. \& Nieuwland, R. Classification, functions, and 
clinical relevance of extracellular vesicles. Pharmacol. Rev. 64, 676-705 (2012).

24. Royo, F. et al. Different EV enrichment methods suitable for clinical settings yield different subpopulations of urinary extracellular vesicles from human samples. J. Extracell. Vesicles 5, 29497 (2016).

25. Wang, J. W. et al. Plasma extra cellular vesicle protein content for diagnosis and prognosis of global cardiovascular disease. Netherlands Hear. J. 21, 467-471 (2013).

26. Urban, S., Mocan, T., Sänger, H., Lukacs-Kornek, V. \& Kornek, M. Extracellular Vesicles in Liver Diseases: Diagnostic, Prognostic, and Therapeutic Application. Semin. Liver Dis. 39, 070-077 (2019).

27. Théry, C. et al. Minimal information for studies of extracellular vesicles 2018 (MISEV2018): a position statement of the International Society for Extracellular Vesicles and update of the MISEV2014 guidelines. J. Extracell. vesicles 7, 1535750 (2018).

28. $\mathrm{Xu}, \mathrm{L}$. et al. Human hepatic stellate cell lines, LX-1 and LX-2: New tools for analysis of hepatic fibrosis. Gut 54, 142-151 (2005).

29. Valentino, G., Zivko, C., Weber, F., Brülisauer, L. \& Luciani, P. Synergy of Phospholipid-Drug Formulations Significantly Deactivates Profibrogenic Human Hepatic Stellate Cells. Pharmaceutics 11, 676 (2019).

30. Bradshaw, A. D. \& Sage, E. H. SPARC, a matricellular protein that functions in cellular differentiation and tissue response to injury. J. Clin. Invest. 107, 1049-1054 (2001).

31. Barker, T. H. et al. SPARC regulates extracellular matrix organization through its modulation of integrin-linked kinase activity. J. Biol. Chem. 280, 36483-36493 (2005).

32. Peixoto, E. et al. SPARC (secreted protein acidic and rich in cysteine) knockdown protects mice from acute liver injury by reducing vascular endothelial cell damage. Gene Ther. 22, 9-19 (2015).

33. Gundermann, K. J., Gundermann, S., Drozdzik, M. \& Mohan Prasad, V. G. Essential phospholipids in fatty liver: A scientific update. Clin. Exp. Gastroenterol. 9, 105-117 (2016).

34. Varganova, D. L., Pavlov, C. S., Casazza, G., Nikolova, D. \& Gluud, C. Essential phospholipids for people with non-alcoholic fatty liver disease (Protocol). Cochrane Database Syst. Rev. 2019, (2019).

35. Dajani, A. I. M. et al. Essential phospholipids as a supportive adjunct in the management of patients with NAFLD. Arab J. Gastroenterol. 16, 99-104 (2015).

36. Sitar, S. et al. Size Characterization and Quantification of Exosomes by Asymmetrical-Flow FieldFlow Fractionation. Anal. Chem. 87, 9225-9233 (2015).

37. Zhang, H. \& Lyden, D. Asymmetric-flow field-flow fractionation technology for exomere and small extracellular vesicle separation and characterization. Nat. Protoc. 14, 1027-1053 (2019).

38. Wu, B. et al. Separation and characterization of extracellular vesicles from human plasma by asymmetrical flow field-flow fractionation. Anal. Chim. Acta 1127, 234-245 (2020).

39. Zhang, $\mathrm{H}$. et al. Identification of distinct nanoparticles and subsets of extracellular vesicles by asymmetric flow field-flow fractionation. Nat. Cell Biol. 20, 332-343 (2018). 
40. Cavallaro, S. et al. Label-Free Surface Protein Profiling of Extracellular Vesicles by an Electrokinetic Sensor. ACS Sensors 4, 1399-1408 (2019).

41. Johann, C., Elsenberg, S., Schuch, H. \& Rösch, U. Instrument and method to determine the electrophoretic mobility of nanoparticles and proteins by combining electrical and flow field-flow fractionation. Anal. Chem. 87, 4292-4298 (2015).

42. Choi, J., Fuentes, C., Fransson, J., Wahlgren, M. \& Nilsson, L. Separation and zeta-potential determination of proteins and their oligomers using electrical asymmetrical flow field-flow fractionation (EAF4). J. Chromatogr. A 1633, 461625 (2020).

43. Dobie, R. et al. Single-Cell Transcriptomics Uncovers Zonation of Function in the Mesenchyme during Liver Fibrosis. Cell Rep. 29, 1832-1847.e8 (2019).

44. Banales, J. M. et al. Extracellular Vesicles in Liver Diseases: Meeting Report from the International Liver Congress 2018. Hepatol. Commun. 3, 305-315 (2019).

45. Meng, W. et al. Prospects and challenges of extracellular vesicle-based drug delivery system: considering cell source. Drug Deliv. 27, 585-598 (2020).

46. Thomas, P. D. et al. PANTHER: A library of protein families and subfamilies indexed by function. Genome Res. 13, 2129-2141 (2003).

47. Mi, H. et al. Protocol Update for large-scale genome and gene function analysis with the PANTHER classification system (v.14.0). Nat. Protoc. 14, 703-721 (2019).

48. Bateman, A. et al. UniProt: A hub for protein information. Nucleic Acids Res. 43, D204-D212 (2015).

49. Larssen, P. et al. Tracing cellular origin of human exosomes using multiplex proximity extension assays. Mol. Cell. Proteomics 16, 502-511 (2017).

50. Fraunhofer, W. \& Winter, G. The use of asymmetrical flow field-flow fractionation in pharmaceutics and biopharmaceutics. Eur. J. Pharm. Biopharm. 58, 369-383 (2004).

51. Bria, C. R. M. \& Williams, S. K. R. Impact of asymmetrical flow field-flow fractionation on protein aggregates stability. J. Chromatogr. A 1465, 155-164 (2016).

52. Citkowicz, A. et al. Characterization of virus-like particle assembly for DNA delivery using asymmetrical flow field-flow fractionation and light scattering. Anal. Biochem. 376, 163-172 (2008).

53. Frank, J. et al. Extracellular vesicles protect glucuronidase model enzymes during freeze-drying. Sci. Rep. 8, 12377 (2018).

54. Oliveira-Rodríguez, M. et al. Development of a rapid lateral flow immunoassay test for detection of exosomes previously enriched from cell culture medium and body fluids. J. Extracell. Vesicles 5, 31803 (2016).

55. Pols, M. S. \& Klumperman, J. Trafficking and function of the tetraspanin CD63. Exp. Cell Res. 315, 1584-1592 (2009).

56. Perez-Hernandez, D. et al. The intracellular interactome of tetraspanin-enriched microdomains reveals their function as sorting machineries toward exosomes. J. Biol. Chem. 288, 11649-11661 (2013). 
57. Bissig, C. \& Gruenberg, J. ALIX and the multivesicular endosome: ALIX in Wonderland. Trends Cell Biol. 24, 19-25 (2014).

58. Thane, K. E., Davis, A. M. \& Hoffman, A. M. Improved methods for fluorescent labeling and detection of single extracellular vesicles using nanoparticle tracking analysis. Sci. Rep. 9, 1-13 (2019).

59. Momen-Heravi, F. et al. Increased number of circulating exosomes and their microRNA cargos are potential novel biomarkers in alcoholic hepatitis. J. Transl. Med. 13, 261 (2015).

60. Hoshino, A. et al. Extracellular Vesicle and Particle Biomarkers Define Multiple Human Cancers. Cell 182, 1044-1061.e18 (2020).

61. Welsh, J. A. et al. MIFlowCyt-EV: a framework for standardized reporting of extracellular vesicle flow cytometry experiments. J. Extracell. Vesicles 9, 1713526 (2020).

62. Gardiner, C. et al. Techniques used for the isolation and characterization of extracellular vesicles: Results of a worldwide survey. J. Extracell. Vesicles 5, 32945 (2016).

63. Freeman, C. M. et al. Characterization of microparticles after hepatic ischemia-reperfusion injury. PLoS One 9, e97945 (2014).

64. Schulz, E., Karagianni, A., Koch, M. \& Fuhrmann, G. Hot EVs - How temperature affects extracellular vesicles. Eur. J. Pharm. Biopharm. 146, 55-63 (2020).

65. Sáenz-Cuesta, M. et al. Methods for extracellular vesicles isolation in a hospital setting. Front. Immunol. 6, 50 (2015).

66. Dragovic, R. A. et al. Sizing and phenotyping of cellular vesicles using Nanoparticle Tracking Analysis. Nanomed.: Nanotechnol. Biol. Med. 7, 780-788 (2011).

67. Dragovic, R. A. et al. Isolation of syncytiotrophoblast microvesicles and exosomes and their characterisation by multicolour flow cytometry and fluorescence Nanoparticle Tracking Analysis. Methods 87, 64-74 (2015).

68. Atorrasagasti, C. et al. Lack of the Matricellular Protein SPARC (Secreted Protein, Acidic and Rich in Cysteine) Attenuates Liver Fibrogenesis in Mice. PLoS One 8, e54962 (2013).

69. Trombetta-eSilva, J. The Function of SPARC as a Mediator of Fibrosis. Open Rheumatol. J. 6, 146155 (2012).

70. Camino, A. M. et al. Adenovirus-mediated inhibition of SPARC attenuates liver fibrosis in rats. J. Gene Med. 10, 993-1004 (2008).

71. Wang, J., Ding, Y. \& Zhou, W. Albumin self-modified liposomes for hepatic fibrosis therapy via SPARC-dependent pathways. Int. J. Pharm. 574, 118940 (2020).

72. Onorato, A. et al. SPARC inhibition accelerates NAFLD-associated hepatocellular carcinoma development by dysregulating hepatic lipid metabolism. Liver Int. 41, 1677-1693 (2021).

73. Zhong, M. E. et al. Serum extracellular vesicles contain SPARC and LRG1 as biomarkers of colon cancer and differ by tumour primary location. EBioMedicine 50, 211-223 (2019).

74. Mallawaaratchy, D. M. et al. Comprehensive proteome profiling of glioblastoma-derived extracellular vesicles identifies markers for more aggressive disease. J. Neurooncol. 131, 233-244 (2017). 
75. Riches, A., Campbell, E., Borger, E. \& Powis, S. Regulation of exosome release from mammary epithelial and breast cancer cells-A new regulatory pathway. Eur. J. Cancer 50, 1025-1034 (2014).

76. Shabbir, A., Cox, A., Rodriguez-Menocal, L., Salgado, M. \& Van Badiavas, E. Mesenchymal Stem Cell Exosomes Induce Proliferation and Migration of Normal and Chronic Wound Fibroblasts, and Enhance Angiogenesis in Vitro. Stem Cells Dev. 24, 1635-1647 (2015).

77. Morelli, A. E. et al. Endocytosis, intracellular sorting, and processing of exosomes by dendritic cells. Blood 104, 3257-3266 (2004).

78. Takov, K., Yellon, D. M. \& Davidson, S. M. Confounding factors in vesicle uptake studies using fluorescent lipophilic membrane dyes. J. Extracell. Vesicles 6, 1388731 (2017).

79. Dehghani, M., Gulvin, S. M., Flax, J. \& Gaborski, T. R. Systematic Evaluation of PKH Labelling on Extracellular Vesicle Size by Nanoparticle Tracking Analysis. Sci. Rep. 10, 1-10 (2020).

80. Gangadaran, P., Hong, C. M. \& Ahn, B. C. An update on in vivo imaging of extracellular vesicles as drug delivery vehicles. Front. Pharmacol. 9, 169 (2018).

81. Richter, M., Fuhrmann, K. \& Fuhrmann, G. Evaluation of the Storage Stability of Extracellular Vesicles. J. Vis. Exp. 147, e59584 (2019).

82. Rahnfeld, L., Thamm, J., Steiniger, F., van Hoogevest, P. \& Luciani, P. Study on the in situ aggregation of liposomes with negatively charged phospholipids for use as injectable depot formulation. Colloids Surf. B Biointerfaces 168, 10-17 (2018).

83. Lydic, T. A. et al. Rapid and comprehensive 'shotgun' lipidome profiling of colorectal cancer cell derived exosomes. Methods 87, 83-95 (2015).

84. Heller, M., Schlappritzi, E., Stalder, D., Nuoffer, J. M. \& Haeberli, A. Compositional protein analysis of high density lipoproteins in hypercholesterolemia by shotgun LC-MS/MS and probabilistic peptide scoring. Mol. Cell. Proteomics 6, 1059-1072 (2007).

85. Braga-Lagache, S. et al. Robust label-free, quantitative profiling of circulating plasma microparticle (MP) associated proteins. Mol. Cell. Proteomics 15, 3640-3652 (2016).

86. Tyanova, S., Temu, T. \& Cox, J. The MaxQuant computational platform for mass spectrometrybased shotgun proteomics. Nat. Protoc. 11, 2301-2319 (2016).

87. Cox, J. et al. Andromeda: A peptide search engine integrated into the MaxQuant environment. J. Proteome Res. 10, 1794-1805 (2011).

88. Hoppstädter, J. et al. Toll-Like Receptor 2 Release by Macrophages: An Anti-inflammatory Program Induced by Glucocorticoids and Lipopolysaccharide. Front. Immunol. 10, 1634 (2019). 


\section{Methods}

\section{Lipid vesicles preparation.}

Liposomal formulations with soybean phospholipids with 75\% phosphatidylcholine (S80, Lipoid) or pure phospholipids 1,2-dioleoyl-sn-glycero-3-phosphocholine (DOPC, Lipoid) were prepared by thin film hydration as previously described ${ }^{29}$. Briefly, extruded 10 times through a $0.2 \mu \mathrm{m}$ polycarbonate membrane (Sterlitech). The hydrodynamic diameter and the size distribution (polydispersity index, PDI) of the liposomes were measured with a Litesizer 500 (Anton Paar).

\section{Cell culture.}

LX-2 cells were grown in high glucose (4'500 mg/L) DMEM (Carl Roth) supplemented with $200 \mathrm{mM} \mathrm{L-}$ Glutamine (Sigma), 10'000 units/L of penicillin and streptomycin (Gibco), and $2 \%$ (v/v) of sterile filtered $(0.2$ $\mu \mathrm{m}$, cellulose acetate membrane) fetal bovine serum (FBS, Merck Millipore).

For experiments, $1.2 \times 10^{6} \mathrm{LX}-2$ were seeded in T175 cell culture flasks and cultured for $120 \mathrm{~h}$, or $1 \times 10^{5}$ cells/well were seeded in 12-well microtiter plates and cultured for $24 \mathrm{~h}$. Cells were then washed with phosphate buffered saline (PBS) and treated for $24 \mathrm{~h}$ with different solutions prepared in serum free cell culture media (DMEM): either ROL/PA (10/300 $\mu \mathrm{M})$, TGF $(10 \mathrm{ng} / \mathrm{mL})$, or liposomal formulations (5 mM lipid concentration) of S80 or DOPC.

\section{EV isolation and purification.}

LX-2 cells were treated in serum free conditions for $24 \mathrm{~h}$ and the CCM from two T175 flasks per treatment $(2 \times 25 \mathrm{~mL})$ was collected (CCMa, which includes treatment solutions). Cells were then washed with PBS and, regardless of previous treatment, they were all supplied with fresh serum free medium (DMEM). After $24 \mathrm{~h}$, the CCM was collected again ( $\mathrm{CCMb}$ ) and cells were split to determine their number and viability. CCMs were centrifuged $\left(300 \times \mathrm{g}, 3 \mathrm{~min}, 4^{\circ} \mathrm{C}\right)$, the supernatant was moved into a new tube and centrifuged again $\left(9^{\prime} 000 \times \mathrm{g}, 30 \mathrm{~min}, 4^{\circ} \mathrm{C}\right)$. The pelleted cell debris was discarded and the supernatant was ultracentrifuged $\left(120^{\prime} 000 \times \mathrm{g}, 2 \mathrm{~h} 30 \mathrm{~min}, 4^{\circ} \mathrm{C}\right)$. After discarding the supernatant, the EV-containing pellet was re-suspended in $0.5 \mathrm{~mL}$ of PBS and purified by size exclusion chromatography (Sepharose ${ }^{\circledR}$ CL-2B, Merck).

All of this is summarized in the following scheme. 


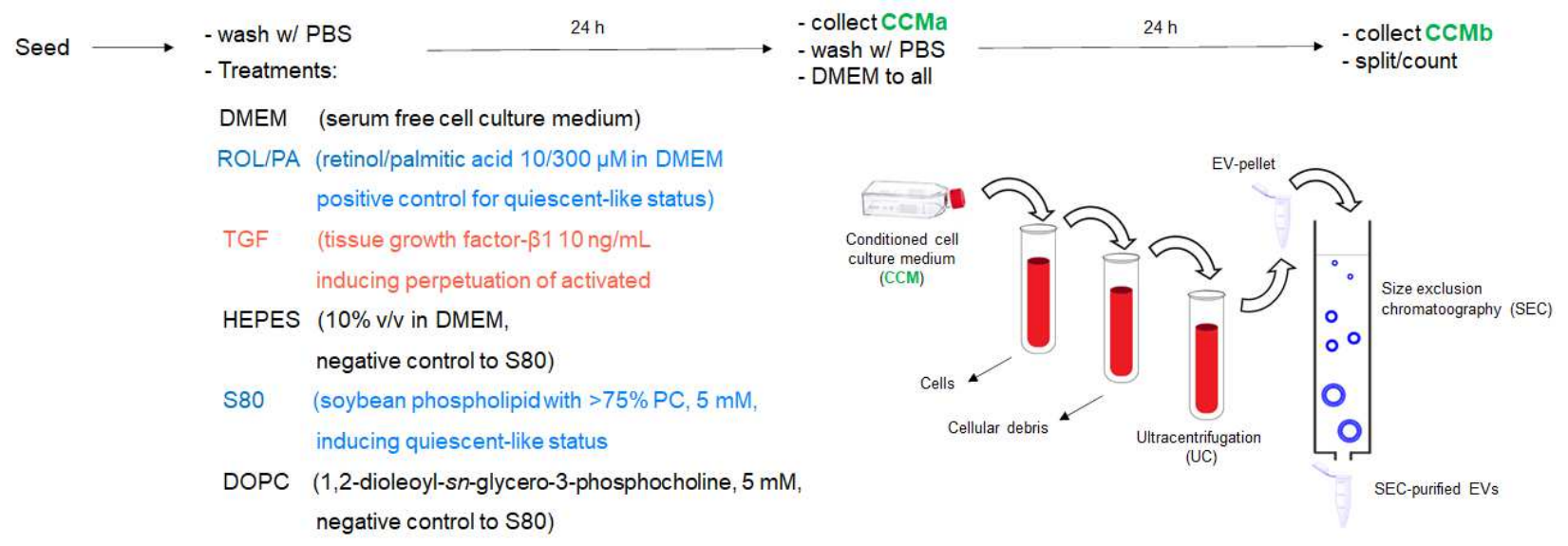

Scheme 1: Overview of the EV isolation and purification protocols.

The collected SEC fractions were analyzed for protein content by means of bicinchoninic acid (BCA) assay. Particle yield, size distribution profiles and zeta potential were determined by nanoparticle tracking analysis (NTA, ZetaView 8.05.05 SP2 equipped with a $488 \mathrm{~nm}$ laser, zetapotential and temperature control units, Particle Metrix). Measurements were performed at $25^{\circ} \mathrm{C}$, a camera sensitivity of 80 , and $100 \mathrm{~ms}^{-1}$ shutter value. Particles were traced for at least 15 consecutive frames, videos were taken at 11 positions. Samples had to have $\geq 200$ traced particles.

Results from the single SEC fractions were consolidated to obtain an average yield and an average particle size, as well as a combined size distribution profile for EVs originating from the differently treated LX-2. Unless otherwise stated, the presented results refer to EVs isolated from CCMb. Every sample was freshly analyzed on the day it was collected, except for those used for electron microscopy imaging, which had been previously stored at $-80{ }^{\circ} \mathrm{C}^{53,81}$.

EV-yield and size distribution profiles have been checked on EVs stored for up to $21 \mathrm{~d}$ under different conditions: $-80{ }^{\circ} \mathrm{C},-25^{\circ} \mathrm{C}, 4{ }^{\circ} \mathrm{C}, 37^{\circ}$ and at room temperature after being freeze-dried with $1 \%$ trehalose $(w / v)^{81}$.

\section{SEM and cryo-TEM}

For scanning electron microscopy (SEM), $10 \mu \mathrm{L}$ re-suspended EV samples were left to dry overnight on silica wafers; they were then sputter-coated with a thin layer of gold for imaging under high vacuum with an accelerating voltage of $5 \mathrm{kV}$ using a Zeiss EVO (Zeiss EVO MA15 LaB6, Oberkochen, Germany) instrument. SEM was performed with the help of Dr. Chiara De Rossi (Helmholtz Institute for Pharmaceutical Research, Saarbrücken).

For cryogenic transmission electron microscopy (cryo-TEM), samples were prepared as previously described ${ }^{22}$. Briefly, $5 \mu \mathrm{L}$ of EV sample were transferred to a copper grid covered by holey carbon film (R1/2, 300 mesh, Quantifoil Micro Tools) and excess liquid was blotted between two strips of filter paper. Samples were plunged into liquid ethane $\left(180^{\circ} \mathrm{C}\right)$ in a cryobox and they were rapidly moved with a Gatan 
626 cryo-transfer holder into the pre-cooled cryo-electron microscope (Philips CM 120) operated at $120 \mathrm{kV}$. The Images were acquired with a $2 \mathrm{k}$ CMOS Camera. Cryo-TEM imaging was performed by Dr. Jana Tamm (Friedrich Schiller University, Jena).

\section{EAF4}

EV pellets from ultracentrifugation were resuspended in approximately $550 \mu \mathrm{L}$ of DMEM. Volumes of 100 $\mu \mathrm{L}$ were injected from a pump and autosampler (Agilent Technologies Germany), sample tray cooled to 8 ${ }^{\circ} \mathrm{C}$, with an Eclipse Dualtec (Wyatt Technologies Europe) equipped with a Mobility EAF4 (Wyatt), a UV absorbance detector (Agilent), and a multi-angle light scattering (MALS) detector Dawn Heleos (Wyatt) for particle detection and size measurement. The Mobility channel was prepared with a narrow spacer (250 $\mu \mathrm{m})$ and contained a $30 \mathrm{kDa}$ molecular weight cut-off regenerated cellulose membrane, which was equilibrated with six injections of cell culture supernatant with $10 \mathrm{mM}$ phosphate buffer $\mathrm{pH} 7.4$ as mobile phase and a detector flow rate of $1 \mathrm{~mL} / \mathrm{min}$. After an equilibration of $1 \mathrm{~min}$ in focus mode with $1.5 \mathrm{~mL}$ crossflow, the sample was injected in focus mode for $5 \mathrm{~min}$, then eluted for $20 \mathrm{~min}$ at $0.2 \mathrm{~mL}$ cross-flow followed by a linear decrease over $5 \mathrm{~min}$ to $0.03 \mathrm{~mL} / \mathrm{min}$ cross-flow and held for $10 \mathrm{~min}$. This was followed by a wash out phase at $0 \mathrm{~mL} / \mathrm{min}$ cross-flow and an elution inject step. Different amperages ranging from +2 to $-6 \mathrm{~mA}$ were applied during the elution with cross-flow phase in consecutive runs. For preparative fractionation the same hardware and membrane were used with a narrow spacer $(350 \mu \mathrm{m})$ and the focus inject time was increased to $8 \mathrm{~min}$ to accommodate and focus the injection volume of $500 \mu \mathrm{L}$ in the channel. Samples were collected at $1 \mathrm{~mL}$ per fraction with an automated fraction collector (Agilent), which was set to the respective sample peaks. Collection times for peak 1 and 2 were as follows: 14.5-18.5 min and 33.5-38.5 min for DMEM, TGF, and DOPC, 21.5-25.5 min and 34.5-42.5 min for ROLPA, 15.5-19.5 min and 34.5-42.5 min for HEPES, and 13.5-19.5 min and 30.5-37.5 min for S80-EVs.

\section{Treatment of fresh LX-2 with EVs isolated from differently treated LX-2.}

EVs produced by LX-2 cells in T175 flasks were isolated from serum free conditioned cell culture medium (CCM) after $24 \mathrm{~h}$ of treatment (CCMa) by differential centrifugation followed by an ultracentrifugation step (see above). Cells were then washed with PBS and given fresh serum-free DMEM. EVs were isolated again after $24 \mathrm{~h}(\mathrm{CCMb})$. EV-containing pellets originating from CCMa and CCMb were re-suspended after UC in fresh, serum-free DMEM, and they were then used to treat LX-2 cells seeded in 12-well plates the day before for $24 \mathrm{~h}$, as well as to treat cells that were see. The presence of cytosolic lipid droplets was determined by Oil Red-O (ORO) staining as previously reported and briefly described below.

\section{Analysis of lipid droplet content - ORO staining.}

After cell treatment, LX-2 in 12-well plates were washed with PBS, fixed with Roti ${ }^{\circledR}$-Histofix and stained with a $0.5 \%$ (w/v) ORO solution in propylene glycol. Nuclei were counterstained with DAPI. Fluorescence and phase contrast images acquisition was performed using a Ti-U inverted microscope (Nikon). The 
quantification of the ORO-stained, fluorescent binary area was normalized to the cell count as determined by DAPI-stained nuclei within the image after thresholding (Fig. S3). For every condition, a total of at least 27 images were acquired: 3 images/well, from 3 separate wells, repeated with 3 biologically independent replicates (performed on separate days from different cellular splits).

\section{Proteomic profiling of EVs.}

SEC-purified EVs, as well AF4-fractioned samples, were transferred to a pre-conditioned polyvinylidene fluoride (PVDF) membrane. First, the PVDF membrane was cut into uniform discs, and the pieces were wetted with methanol $(\mathrm{MeOH})$ for $5 \mathrm{~min}$. After removal of the $\mathrm{MeOH}$, a solution of $0.05 \%$ sodium dodecyl sulfate (SDS)/5\% MeOH/0.05\% Dithiothreitol (DTT) was added for 5 min. Membrane pieces were placed into the bottom of the collection tubes right before EV collection with a little PBS to keep them wet. After the purification step (either by SEC or AF4), the PVDF membranes and EV-containing samples were centrifuged (3'000 x g, $1 \mathrm{~h}, \mathrm{RT}$ ), and the supernatant discarded. PVDF membrane pieces were dried under $\mathrm{N}_{2}$ flow for $15 \mathrm{~min}$ and stored at $4{ }^{\circ} \mathrm{C}$. Comparative, shotgun proteomics was performed after reductive alkylation and trypsin digestion of the samples ${ }^{83,84}$ by the Proteomics Mass Spectrometry Core Facility (PMSCF) at the Department of Biomedical Research (DBMR) of the University of Bern. Peptides were analyzed by liquid chromatography tandem mass spectrometry (nano-LC-MS/MS, ThermoFisher Scientific) and spectra were searched by MaxQuant/Andromeda ${ }^{85-87}$.

\section{Staining of EVs with PKH67.}

SEC-purified EVs were incubated with 1.5 - $24 \mu \mathrm{M}$ of PKH67 (Merck) for $5-120$ min at $24{ }^{\circ} \mathrm{C}$ under gentle shaking. NTA-measurements were performed in scatter mode as previously described (see above), but the sensitivity was changed to 90 for measurements in fluorescence mode (f-NTA).

\section{Detection of exosomal marker CD9 by flow cytometry.}

The analysis of EVs by flow cytometry (FACS) was performed as previously reported ${ }^{88}$. Briefly, EVs were coupled to $4 \mu \mathrm{m}$ aldehyde/sulfate latex beads. EV-containing SEC-fractions ( $1 \mathrm{~mL}$ each, see above) were divided into $0.5 \mathrm{~mL}$ aliquots. Freshly filtered (CA, $200 \mathrm{~nm}) \mathrm{BSA} 1 \% \mathrm{w} / \mathrm{v}$ in PBS was also prepared as a negative control. All samples were then incubated for 15 min at RT with $10 \mu \mathrm{L}$ of latex beads. PBS was added up to $1 \mathrm{~mL}$ and all samples were incubated for $1 \mathrm{~h}$ at RT with gentle shaking. The reaction was stopped with $0.5 \mathrm{~mL}$ of $200 \mathrm{mM}$ glycine, incubated for $30 \mathrm{~min}$ at RT. Beads coupled to EVs (or BSA) were centrifuged (2'000 x g, 3 min, RT), the supernatant was removed and the pelleted beads were re-suspended with BSA $1 \% \mathrm{w} / \mathrm{v}$ in PBS. This washing step was repeated two more times. Samples were stained with fluorescently labeled antibodies for CD9 (6 ng/mL, IgG2b, Clone \#209306) or with the fluorescently labeled isotype control (IC) in ice and in the dark for 30 min. Finally, samples were washed twice with BSA $1 \%$ in PBS and analyzed on a BD LRS Fortessa (BD Biosciences) using BD FACSDiva 8.0. 


\section{Antibody labelling.}

Antibodies against human CD81 (IgG2B, Clone \#454720, Biotechne) and SPARC (IgG1, Clone \#122511, Biotechne) were conjugated with AlexaFluor488 (AF488) using the Lighting-Link ${ }^{\circledR}$ (LL) antibody labelling kit (Biotechne) as per manufacturer's instructions and under sterile conditions. Briefly, the LL-modifier solution was added to the unconjugated antibody in sterile PBS $(1 \mu \mathrm{L}$ for every $10 \mu \mathrm{L}$ of antibody). This was then used to re-suspend the lyophilized mixture with AF488, and left incubating for $15 \mathrm{~min}$ at RT. LL-quencher was then added to the antibody-AF488 mixture ( $1 \mu \mathrm{L}$ for every $10 \mu \mathrm{L}$ of antibody). The LL-kit was also used to prepare an isotype control with the IgG chain (Goat anti-Human IgG $(H+L)$, Cross-Adsorbed Secondary Antibody, ThermoFisher Scientific). The performance on EVs of AF488-CD81 already conjugated at purchase and LL-conjugated AF488-CD81 were compared as well (Fig. S17).

\section{Detection of EV-associated proteins with f-NTA.}

Incubation with AlexaFluor488 conjugated antibodies for exosomal marker CD81 (AF488-CD81) was performed directly into SEC-purified samples for different times (every $10 \mathrm{~min}$ up to $120 \mathrm{~min}$, every hour for up to $6 \mathrm{~h}$ and then overnight), at different temperatures (in ice, at 24 and $37^{\circ} \mathrm{C}$ ), with different concentrations (0.05 - $1 \mathrm{ng} / \mathrm{mL})$. Only data with successful f-NTA detection could be shown.

Incubation with AF488-CD81, LL-AF488-CD81, AF488-CD9, LL-AF488-SPARC, were performed in the resuspended EV-containing pellets obtained after UC, prior to SEC. The protocol optimization was done with AF488-CD81. The tested conditions included different incubation temperatures (24 and $37^{\circ} \mathrm{C}$ ), for variable amounts of time (for 3 or $5 \mathrm{~h}$ ), using $2-8 \mathrm{ng} / \mathrm{mL}$ of antibody conjugates.

Measurements were performed in scatter mode as previously described (see above), but the sensitivity was changed to 90 for measurements in fluorescence mode. For every protocol yielding a measurable result, incubation with isotype controls (either bought already conjugated, or LL-conjugated) were also performed.

For antibody incubations with EV-pellets originating from differently treated LX-2 cells, the regimens were as follows: DMEM, ROL/PA (10/300 $\mu \mathrm{M})$, TGF (10 ng/mL), HEPES buffer (10\% v/v), S80 (5 mM), DOPC (5 mM). The labeled EVs were then purified and collected after SEC. 


\section{Supplementary Files}

This is a list of supplementary files associated with this preprint. Click to download.

- ZivkoSI.pdf

- 2021czivkor01proteins.xlsx 